\title{
Optimal control in laser-induced population transfer for two- and three-level quantum systems
}

\author{
Ugo Boscain ${ }^{\text {a) }}$ \\ Département de Mathématiques, Analyse Appliquée et Optimisation, Université de \\ Bourgogne, 9, Avenue Alain Savary, B.P. 47870-21078 Dijon Cedex, France \\ Grégoire Charlot ${ }^{\mathrm{b})}$ \\ Laboratoire GTA, Case Courrier 51, Université Montpellier II, \\ 34095 Montpellier Cedex 5, France \\ Jean-Paul Gauthier ${ }^{\mathrm{c})}$ \\ Département de Mathématiques, Analyse Appliquée et Optimisation, Université de \\ Bourgogne, 9, Avenue Alain Savary, B.P. 47870-21078 Dijon Cedex, France \\ Stéphane Guérin ${ }^{\text {d) }}$ and Hans-Rudolf Jausline) \\ Laboratoire de Physique de l'Université de Bourgogne, 9, Avenue Alain Savary, \\ B.P. 47870-21078 Dijon Cedex, France
}

(Received 19 October 2001; accepted for publication 23 January 2002)

\begin{abstract}
We apply the techniques of control theory and of sub-Riemannian geometry to laser-induced population transfer in two- and three-level quantum systems. The aim is to induce complete population transfer by one or two laser pulses minimizing the pulse fluences. Sub-Riemannian geometry and singular-Riemannian geometry provide a natural framework for this minimization, where the optimal control is expressed in terms of geodesics. We first show that in two-level systems the wellknown technique of " $\pi$-pulse transfer" in the rotating wave approximation emerges naturally from this minimization. In three-level systems driven by two resonant fields, we also find the counterpart of the " $\pi$-pulse transfer." This geometrical picture also allows one to analyze the population transfer by adiabatic passage. (C) 2002 American Institute of Physics. [DOI: 10.1063/1.1465516]
\end{abstract}

\section{INTRODUCTION: PHYSICAL CONTEXT}

\section{A. Generalities}

Design of external laser fields (amplitudes and frequencies) to reach a selected state of a quantum system is of primary importance for the control of quantum dynamics. The techniques for this state-selectivity that have been developed are essentially based on (i) adiabatic passage (see, e.g., the recent works ${ }^{1-4}$ ), (ii) multiphoton quasiresonant pumping ${ }^{5}$ by "generalized $\pi$-pulses," 6 and (iii) optimal control theory (see, e.g., Refs. 7 and 8).

Adiabatic passage has the advantage of robustness in the sense that significant deviations of the fields do not significantly modify the final result. On the other hand, optimal control is a systematic framework to design the field parameters (or control variables) to reach selectivity in maximizing or minimizing a quantity (the cost) depending functionally on the state and control variables. This design is in general not robust, in contrast with standard adiabatic passage. Since the robustness is difficult to quantify as a cost, we do not expect in general the adiabatic passage to emerge from an optimal control solution. This has been discussed for specific simple systems. ${ }^{9,10}$ However optimality for adiabatic passages has been characterized in Ref. 11.

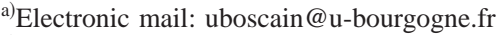

b) Electronic mail: charlot@math.univ-montp2.fr

${ }^{c}$ Electronic mail: gauthier@u-bourgogne.fr

d)Electronic mail: Stephane.Guerin@u-bourgogne.fr

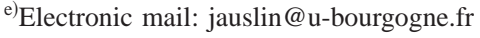


The success of these coherent laser-induced processes requires, in general, use of as short as possible external pulses, to minimize incoherent effects of relaxation (through spontaneous emission, collisions, etc.). It is essential that the total time of the pulse is shorter than the characteristic times of the incoherent losses. Coherent transfers also need as low as possible pulse intensities in order to (i) minimize incoherent phenomena induced by strong fields such as ionization for atoms or molecules, and (ii) avoid involving other energy levels, that are not included in the models. Otherwise the population is spread among these levels and the transfer becomes inefficient.

Depending on the concrete physical setting there are several possible choices of the cost functional. One choice, which has been studied in Ref. 12, is to minimize the total time of the control process. However, the total time minimization presented in Ref. 12 assumes that arbitrarily large laser intensities can be used. The relations between our results and the results of Ref. 12 will be made clear in Sec. II C.

In this paper we address the two- and three-level problem from the geometric control theory point of view. We consider several functionals to be minimized, which are geometrically very natural, and that physically reflect the practical constraints mentioned previously [fluence, see formula (5) for the two-level case and formula (7) for the three-level case, or transfer time with bounded controls, etc.]. The choice of these costs will be discussed in Sec. II C. We will also use the classical tracking technique, often used in in geometric control theory (see Ref. 13), that will allow in particular achievement of adiabatic transfer.

Remark 1: In the choice of the cost to be minimized, it is useful to consider optimal problems that are independent of time reparametrization. Indeed, in that case, very slowly varying solutions, comparable with the ones used in experiments, can be obtained just by time reparametrization. This point will be taken into account in the following, in particular see Sec. II C. But we will also consider problems that do not have this feature.

\section{B. Content of the paper}

In this work we apply geometric control theory in two- and three-level systems, which we briefly describe in the following. Moreover we make the connection with the adiabatic passage.

\section{The two-level case}

We first study the population transfer in a two-level quantum system (of energies $E_{1}$ and $E_{2}$ ) driven by an external field of arbitrary time-dependent shape, starting at $t_{0}$ and ending at $t_{1}$. The dynamics is governed by the time-dependent Schrödinger equation (in a system of units such that $\hbar=1)$ :

$$
i \frac{\mathrm{d} \psi(t)}{\mathrm{d} t}=H \psi(t),
$$

$\psi():. \mathbb{R} \rightarrow \mathbb{C}^{n}, n=2$, where

$$
H=\left(\begin{array}{cc}
E_{1} & \Omega(t) \\
\Omega^{*}(t) & E_{2}
\end{array}\right) .
$$

is the Hamiltonian of the system (we have assumed no diagonal coupling). We choose the most general situation where the off-diagonal element is complex: $\Omega(t): \mathbb{R} \rightarrow \mathrm{C}$.

In the two-level system (2), complete controllability (on spheres) is obvious (see the following for the precise definition of controllability). We show that the natural optimal control problem of minimizing the integral of the laser amplitude:

$$
\ell=\int_{t_{0}}^{t_{1}}|\Omega| \mathrm{d} t
$$

has the following features. 
(a) After some transformations the problem can be formulated as a problem of SubRiemannian geometry (see Remark 3).

(b) The optimal control solution has the expected resonance property, i.e., $\Omega$ can be written as

$$
\Omega(t)=b(t) e^{i\left[\left(E_{2}-E_{1}\right) t+\alpha\right]},
$$

where $\alpha$ is an arbitrary constant, $b():. \mathbb{R} \rightarrow \mathbb{R}^{+}$is a real function with compact support $\left[t_{0}, t_{1}\right]$.

(c) The optimal amplitude $b(t)$ satisfies

$$
\int_{t_{0}}^{t_{1}} b(t)=\pi / 2
$$

Remark 2: We thus recover the well-known strategy of " $\pi$-pulse transfer" of a resonant pulse in the rotating wave approximation, ${ }^{14}$ which gives complete transfer with $\Omega(t)=\mu \mathcal{F}(t) e^{i \phi(t)} / 2$, $\dot{\phi}(t)=E_{2}-E_{1}$, and $\ell=|\mu| \int_{t_{0}}^{t_{1}} \mathcal{F}(t) \mathrm{d} t / 2=\pi / 2$. Here $\mu$ is the intrinsic coupling between the two levels and $\mathcal{F}(t)$ the external pulsed field. This shows in particular that an additional controllable time-dependent frequency ("chirping," see, e.g., Ref. 15) does not improve the minimization with respect to this cost. We thus show that in the model of the rotating wave approximation, the " $\pi$-pulse transfer" corresponding to the minimum pulse area to achieve the complete transfer is a consequence of purely geometric considerations.

(d) The solution is independent of time reparametrization, i.e., derivatives of controls can be made as small as required simply by choosing an appropriate time parametrization. Moreover, with an adequate choice of the parametrization it minimizes the fluence for fixed transfer time $t_{1}-t_{0}$ :

$$
\mathcal{E}=\int_{t_{0}}^{t_{1}}|\Omega|^{2}(t) \mathrm{d} t=\int_{t_{0}}^{t_{1}} b^{2}(t) \mathrm{d} t,
$$

or equivalently the transfer time, with the constraint on the amplitude $|b(t)| \leqslant 1$. More details about the relations between these costs are given in Sec. II C.

Remark 3: A control problem is called distributional if the set of admissible velocities is a distribution, i.e., a nonintegrable field of planes. It is called contact if the distribution is a contact distribution, i.e., if the field of planes is defined as the kernel of the one-form $\omega$, then $\mathrm{d} \omega$ is nondegenerate when restricted to $\operatorname{Ker}(\omega)$. A control problem is said to be sub-Riemannian if additionally one gives a Riemannian metric on the distribution, and one minimizes the Riemannian length. For sub-Riemannian geometry we refer to Refs. 16-18.

In fact, as we shall see, the two-level problem reduces to a three-dimensional contact subRiemannian problem, with a special feature: it has a symmetry, transverse to the distribution. It is a standard fact that such a sub-Riemannian problem is in fact an isoperimetric problem (in the sense of the calculus of variations) on the quotient by the symmetry. Such isoperimetric contact three-dimensional sub-Riemannian problems have been studied in detail at the local level in Ref. 19, for instance. In fact, the above-given statement is nothing but the (trivial) solution of the classical isoperimetric problem (or Dido problem) on the Riemannian sphere.

The study of this two-level case is described in Sec. III.

\section{The three-level case}

For three-level systems, labeled 1,2 , and 3 , of respective energies $E_{1}, E_{2}$, and $E_{3}$, driven by two resonant monochromatic fields of respective envelopes $\mathcal{F}_{j}(t), j=1,2$ and of frequencies $\omega_{1}$ and $\omega_{2}$, the Hamiltonian, in the rotating wave approximation (RWA), reads 


$$
H=\left(\begin{array}{ccc}
E_{1} & u_{1}(t) e^{i \omega_{1} t} & 0 \\
u_{1}(t) e^{-i \omega_{1} t} & E_{2} & u_{2}(t) e^{i \omega_{2} t} \\
0 & u_{2}(t) e^{-i \omega_{2} t} & E_{3}
\end{array}\right),
$$

where

$$
\begin{array}{ll}
\omega_{1}:=E_{2}-E_{1}, & u_{1}(.): \mathbb{R} \rightarrow \mathbb{R}, \\
\omega_{2}:=E_{3}-E_{2}, & u_{2}(.): \mathbb{R} \rightarrow \mathbb{R} .
\end{array}
$$

The controls $u_{j}(t), j=1,2$ are connected to the physical parameters by $u_{j}(t)=\mu_{j} \mathcal{F}_{j}(t) / 2$, with the couplings $\mu_{j}$, intrinsic to the quantum system, that we have restricted to couple only levels $j$ and $j+1$ by pairs. We allow the control variables, starting at $t_{0}$ and ending at $t_{1}$, to have any shape.

Controllability. For a control system, the accessibility set of a point $x_{0}$ in the configuration space is the set of points that can be joined from $x_{0}$ by a trajectory of the system. A control system is said to be controllable if the accessible set of every $x_{0}$ is the whole configuration space. For an analytic distributional system, it is known that the configuration space is foliated by accessible sets, called orbits in that case. These orbits are exactly the Hermann-Nagano orbits (see the Hermann-Nagano Theorem in the following and Ref. 22 for details).

In the following we first prove that this three-level system reduces to a distributional problem which is not controllable, since the orbits under consideration are two-dimensional spheres. Then we show that on each of these spheres the control problem reduces to a singular-Riemannian problem. The "relevant locus," which is the union of all the orbits (spheres) passing through state number 1 , has an interesting nontrivial geometric description. It is the only nonorientable spherebundle over $S^{1}$ (see Sec. IV A).

Optimal solutions. In this case we first consider the problem of minimizing the fluence for fixed transfer time $t_{1}-t_{0}$ (which is a parametrization-dependent cost):

$$
\mathcal{E}=\int_{t_{0}}^{t_{1}}\left(u_{1}^{2}+u_{2}^{2}\right) \mathrm{d} t
$$

Again let us notice that minimization of the functional:

$$
\ell=\int_{t_{0}}^{t_{1}} \sqrt{u_{1}^{2}+u_{2}^{2}} \mathrm{~d} t
$$

leads to the same motion in the space state, parametrized in any way. Moreover, minimizing the functional $\mathcal{E}$ is equivalent to minimizing the transfer-time for controls bounded in the following way:

$$
u_{1}^{2}+u_{2}^{2} \leqslant 1
$$

This will be explained in detail in Sec. IIC.

For the three-level system (6), the main known ways to completely populate state 3 from the initial condition in state 1 are the following:

(i) application of two successive $\pi$-pulses (without overlap), giving $\ell=\pi$ in the system (6);

(ii) interaction with two completely overlapping control variables: $u_{1}(t)=u_{2}(t)$ (with two different pulses assuming that the couplings between 1 and 2 and between 2 and 3 are different), giving also $\ell=\pi ;{ }^{20}$

(iii) adiabatic passage by delayed pulses (of the same shape) such that $u_{2}(t)$ starts before $u_{1}(t)$ (known as "counterintuitive" pulse sequence). ${ }^{1}$ This way of transferring the population is energetically expensive since it requires $\ell \gg \pi$. 
For this three-level system, we show that the cost $\ell$ can be significantly reduced, finding its minimum value

$$
\ell=\frac{\sqrt{3}}{2} \pi \approx 0.866 \pi,
$$

corresponding to the singular-Riemannian geodesic. This is also the minimum transfer time under the constraint (9).

The minimum value for the functional $\mathcal{E}$ is

$$
\mathcal{E}=\frac{3}{4} \pi^{2} \frac{1}{t_{1}-t_{0}}
$$

[where $\left(t_{1}-t_{0}\right)$ is the fixed interaction time]. These results are described by Theorem 4 of Sec. IV.

We show that the associated pulse sequence is such that $u_{1}(t)$ starts before $u_{2}(t)$ ("intuitive" sequence) and we construct symmetric smooth pulses of the same shape giving this minimum. We compute the geodesic joining the initial state to the final state, and we give some examples of time reparametrized optimal controls. The results thus show very natural control strategies that do not look like the standard strategy in this type of problem. This study is the content of Sec. IV.

Tracking. In Sec. V we construct for the three-level problem a geometric representation of adiabatic passage and compare it with the strategies of optimal control. This allows one to reformulate the adiabatic passage as explicitly fixing the trajectory leading from state 1 to state 3 and in controlling the rate of transient population in the intermediate state 2 . This procedure improves on the standard adiabatic passage in that it exactly reaches the target state. This tracking technique is standard in control theory. A solution of this type is generated by a counterintuitive sequence of controls, in the adiabatic approximation. ${ }^{1}$

\section{PRELIMINARIES, ELIMINATION OF THE DRIFT, AND CHOICE OF THE COST}

\section{A. Preliminaries}

We start with a quantum system with finite number of (distinct) levels in interaction with a time-dependent external field such that the Hamiltonian reads:

$$
\begin{gathered}
H=\left(\begin{array}{ccccc}
E_{1} & \Omega_{1}(t) & 0 & \cdots & 0 \\
\Omega_{1}^{*}(t) & E_{2} & \Omega_{2}(t) & \ddots & \vdots \\
0 & \Omega_{2}^{*}(t) & \ddots & \ddots & 0 \\
\vdots & \ddots & \ddots & E_{n-1} & \Omega_{n-1}(t) \\
0 & \cdots & 0 & \Omega_{n-1}^{*}(t) & E_{n}
\end{array}\right) \\
=D+\left(\begin{array}{ccccc}
0 & \Omega_{1}(t) & 0 & \cdots & 0 \\
\Omega_{1}^{*}(t) & 0 & \Omega_{2}(t) & \ddots & \vdots \\
0 & \Omega_{2}^{*}(t) & \ddots & \ddots & 0 \\
\vdots & \ddots & \ddots & 0 & \Omega_{n-1}(t) \\
0 & \cdots & 0 & \Omega_{n-1}^{*}(t) & 0
\end{array}\right),
\end{gathered}
$$

with $D:=\operatorname{diag}\left(E_{1}, \ldots, E_{n}\right)$ and the energies of the system states appearing on the diagonal. Timedependent elements $\Omega_{j}():. \mathrm{R} \rightarrow \mathrm{C}, j=1, \ldots, n-1$ are different from zero only between times $t_{0}$ and $t_{1}$. They couple the states by pairs. The term $D$ is called drift. Section II B is devoted to the elimination of $D$ via a unitary transformation. 
The state-vector $\psi(t)$, solution of the time-dependent Schrödinger equation (1), can be expanded in the canonical basis of $\mathbb{C}^{n}$, formed by elements $\varphi_{1}=(1,0, \ldots, 0), \varphi_{2}$ $=(0,1, \ldots, 0), \ldots \varphi_{n}=(0,0, \ldots, 1): \psi(t)=c_{1}(t) \varphi_{1}+c_{2}(t) \varphi_{2}+\cdots+c_{n}(t) \varphi_{n}$, with $\left|c_{1}(t)\right|^{2}$ $+\left|c_{2}(t)\right|^{2}+\cdots+\left|c_{n}(t)\right|^{2}=1$. For $t<t_{0}$ and $t>t_{1},\left|c_{i}(t)\right|^{2}$ is the probability of measuring energy $E_{i}$. Notice that

$$
\frac{\mathrm{d}}{\mathrm{d} t}\left|c_{i}(t)\right|^{2}=0 \quad \text { for } t<t_{0} \text { and } t>t_{1}
$$

Our problem can be stated in the following way: Assuming $\left|c_{1}(t)\right|^{2}=1$ for $t<t_{0}$, we want to determine suitable interaction functions $\Omega_{i}(),. i=1, \ldots, n-1$, such that $\left|c_{j}(t)\right|^{2}=1$ for time $t$ $>t_{1}$ and some chosen $j \in\{2, \ldots, n\}$, requiring that they minimize the cost (8).

Remark 4: This problem is a control problem on the real $2 n-1$ dimensional sphere in $\mathbb{R}^{2 n}$ (or on the complex sphere in $\left.\mathbb{C}^{n}\right)$. Standard considerations from control theory allow one to conclude on the controllability on the sphere [even with arbitrarily small bounds on the controls $\Omega_{i}($.$) ], i.e.,$ it is possible to drive the system from any initial condition to any terminal condition on the sphere. Also, control problems of this type have finite dimensional Lie-algebra: the reductive Lie algebra $s u(n) \times \mathbb{R}$. They can be lifted to left-invariant control problems on the compact Lie group $\mathrm{SU}(n) \times S^{1}\left(S^{1}\right.$, the circle), and the controllability of the lifted control system also holds.

We will also consider the "real resonant case" in which the controls $\Omega_{i}$ correspond to lasers that are in resonance:

$$
\begin{gathered}
\Omega_{i}(t)=u_{i}(t) e^{i \omega_{i} t}, \quad \omega_{i}=E_{i+1}-E_{i}, \\
i=1, \ldots, n-1, \quad u_{i}(.): \mathbb{R} \rightarrow \mathbb{R} .
\end{gathered}
$$

Notice that in this case we consider real controls which always leads to lack of controllability.

In the following we treat the $n=2$ and $n=3$ cases. The $n=2$ case is treated in the most general setting, in the sense that we control both the amplitude and the phase of the laser pulses. We obtain that the optimal strategy is realized with an external pulse in resonance in the rotating wave form. The $n=3$ case is treated directly with pulses in resonance in the rotating wave approximation.

In the $n=3$ case, it is an open question if it is possible to reduce the value of the cost (8), when controlling both amplitude and phase of the lasers.

In the following, to compute the orbits of the control systems under consideration, we will make use of the standard Hermann-Nagano theorem. Moreover to compute optimal trajectories we will use the well-known Pontryagin Maximum Principle. ${ }^{21}$ For convenience we recall these two theorems in the following. Proofs can be found for instance in Ref. 22.

Theorem (Hermann-Nagano): Let $M$ be an analytic manifold and $\mathcal{F}$ a family of analytic vector fields on $M$. Then:

(1) each orbit of $\mathcal{F}$ is an analytic submanifold of $M$,

(2) if $\mathcal{O}\left(\mathbf{x}_{0}\right)$ is an orbit containing the point $\mathbf{x}_{0}$, then the tangent space of $\mathcal{O}\left(\mathbf{x}_{0}\right)$ at $\mathbf{x}$ is given by

$\operatorname{Lie}_{\mathbf{x}}(\mathcal{F})$. In particular the dimension of $\operatorname{Lie}_{\mathbf{x}}(\mathcal{F})$ is constant as $\mathbf{x}$ varies over $\mathcal{O}\left(\mathbf{x}_{0}\right)$.

Theorem (Pontryagin Maximum Principle): Consider a control system of the form $\dot{x}$ $=f(x, u)$ with a cost of the form $\int_{0}^{T} f^{0}(x, u) \mathrm{d} t$, where $x$ belongs to a manifold $M$ and $u$ $\in U \subset \mathbb{R}^{m}$. Assume moreover that $M, f, f^{0}$ are smooth. If the couple $(u(),. x()):.[0, T] \subset \mathbb{R} \rightarrow U$ $\times M$ is optimal, then there exists a never vanishing field of covectors along $x($.$) , that is an$ absolutely continuous function $\left(p(),. p^{0}\right): t \in[0, T] \mapsto p(t) \in T_{x(t)}^{*} M \times \mathbb{R}$ (where $p_{0} \leqslant 0$ is a constant) such that:

(i) $\dot{x}(t)=\partial \mathcal{H} / \partial p(x(t), p(t), u(t))$,

(ii) $\dot{p}(t)=-\partial \mathcal{H} / \partial x(x(t), p(t), u(t))$, 
where by definition

$$
\mathcal{H}(x, p, u):=<p, f(x, u)>+p_{0} f^{0}(x, u) .
$$

Moreover:

(iii) $\mathcal{H}(x(t), p(t), u(t))=\mathcal{H}_{M}(x(t), p(t))$, for a.e. $t \in[0, T]$,

where $\mathcal{H}_{M}(x(t), p(t)):=\max _{v \in U} \mathcal{H}(x(t), p(t), v)$.

The real-valued map on $T^{*} M \times U$, defined in (12), is called Hamiltonian. The couples $(u(),. x()$.$) satisfying conditions (i)-(iii) with p_{0}=0$ are called abnormal extremals.

\section{B. Elimination of the drift term}

In both cases (2) and (6) we show how to eliminate the drift term [i.e., $\operatorname{diag}\left(E_{1}, E_{2}\right)$ and $\operatorname{diag}\left(E_{1}, E_{2}, E_{3}\right)$ respectively] from the Hamiltonian. In case (6) this elimination will be made just by a unitary change of coordinates that at the same time eliminates the explicit dependence on the time. In case (2) it will moreover require a unitary change of controls. This difference is simply a consequence of the fact that in the three-level case we start with "real controls in resonance," while in the two-level case we use general complex controls.

Assume that $\psi(t)$ satisfies the Schrödinger equation (1). Let $U(t)$ be a unitary time-dependent matrix and set $\psi(t)=U(t) \psi^{\prime}(t)$ (interaction representation). Then $\psi^{\prime}(t)$ satisfies the Schrödinger equation:

$$
i \frac{\mathrm{d} \psi^{\prime}(t)}{\mathrm{d} t}=H^{\prime}(t) \psi^{\prime}(t),
$$

with the new Hamiltonian:

$$
H^{\prime}=U^{-1} H U-i U^{-1} \frac{\mathrm{d} U}{\mathrm{~d} t} .
$$

Here we consider the Hamiltonian (10), and we choose

$$
U=\operatorname{diag}\left(e^{-i E_{1} t}, e^{-i E_{2} t}, \ldots, e^{-i E_{n} t}\right) .
$$

We get:

$$
H^{\prime}=\left(\begin{array}{ccccc}
0 & \Omega_{1}(t) e^{-i\left(E_{2}-E_{1}\right) t} & 0 & \cdots & 0 \\
\Omega_{1}^{*}(t) e^{i\left(E_{2}-E_{1}\right) t} & 0 & \Omega_{2}(t) e^{-i\left(E_{3}-E_{2}\right) t} & \ddots & \vdots \\
0 & \Omega_{2}^{*}(t) e^{i\left(E_{3}-E_{2}\right) t} & \ddots & \ddots & 0 \\
\vdots & \ddots & \ddots & 0 & \Omega_{n-1}(t) e^{-i\left(E_{n}-E_{n-1}\right) t} \\
0 & \cdots & 0 & \Omega_{n-1}^{*}(t) e^{i\left(E_{n}-E_{n-1}\right) t} & 0
\end{array}\right) .
$$

As a consequence, if we write $\psi(t)=c_{1}(t) \varphi_{1}+c_{2}(t) \varphi_{2}+\cdots+c_{n}(t) \varphi_{n}$, and $\psi^{\prime}(t)=c_{1}^{\prime}(t) \varphi_{1}$ $+c_{2}^{\prime}(t) \varphi_{2}+\cdots+c_{n}^{\prime}(t) \varphi_{n}$, then $\left|c_{i}(t)\right|^{2}=\left|c_{i}^{\prime}(t)\right|^{2}, i=1,2, \ldots, n$, that is $H$ and $H^{\prime}$ have the same population distribution.

For the three-level system (6), this leads to (dropping the prime)

$$
H=\left(\begin{array}{ccc}
0 & u_{1}(t) & 0 \\
u_{1}(t) & 0 & u_{2}(t) \\
0 & u_{2}(t) & 0
\end{array}\right)
$$

For the two-level system (2), redefining

$$
u(t):=e^{-i\left(E_{2}-E_{1}\right) t} \Omega(t),
$$


we get (dropping the prime)

$$
H=\left(\begin{array}{cc}
0 & u(t) \\
u^{*}(t) & 0
\end{array}\right)
$$

In the following we set $u(t)=u_{1}(t)+i u_{2}(t)$, where $u_{1}($.$) and u_{2}($.$) are two real functions.$

Remark 5: Notice that the unitary transformations on the states and on the controls preserve the cost and the probabilities. As a consequence they preserve the initial and final conditions $\left|c_{1}\left(t_{0}\right)\right|^{2}=1,\left|c_{j}\left(t_{1}\right)\right|^{2}=1$.

Remark 6: This reduction procedure can be easily extended to the case where the drift term in (10) is time-dependent:

$$
D=\operatorname{diag}\left(E_{1}(t), \ldots, E_{n}(t)\right)
$$

The key point is that the couplings have to be only between successive levels. For the two-level case, this requirement is obviously met. The elimination of the drift (18) in (10) requires the matrix

$$
U=\operatorname{diag}\left(\exp \left(-i \int_{t_{0}}^{t} E_{1}(s) \mathrm{d} s\right), \exp \left(-i \int_{t_{0}}^{t} E_{2}(s) \mathrm{d} s\right), \ldots, \exp \left(-i \int_{t_{0}}^{t} E_{n}(s) \mathrm{d} s\right)\right) .
$$

In this case the new Hamiltonian has the form (14) with $i\left(E_{i+1}-E_{i}\right) t$ replaced by $i \int_{t_{0}}^{t}\left(E_{i+1}(s)\right.$ $\left.-E_{i}(s)\right) \mathrm{d} s$ and the resonance condition (11) becomes

$$
\Omega_{i}(t)=u_{i}(t) \exp \left(i \int_{t_{0}}^{t}\left(E_{i+1}(s)-E_{i}(s)\right) \mathrm{d} s\right), \quad i=1, \ldots, n-1, \quad u_{i}(.): \mathbb{R} \rightarrow \mathbb{R} .
$$

\section{Choice of different costs and relation between them}

\section{Minimizing length and energy}

As we will show, both the $n=2$ and $n=3$ problems can be stated as control problems that are linear with respect to the controls (i.e., "distributional control problems"):

$$
\dot{\mathbf{x}}=u_{1} F_{1}+u_{2} F_{2},
$$

where $\mathbf{x} \in S^{3}$ for $n=2$ and $\mathbf{x} \in S^{5}$ for $n=3$, with $F_{1}$ and $F_{2}$ two vector fields on the $d$-dimensional sphere $S^{d}$. It is thus natural to treat this problem as a sub-Riemannian problem (in the three-level case it is in fact singular-Riemannian problem on $S^{2}$, see Sec. IV), to which is associated the length

$$
\ell=\int_{t_{0}}^{t_{1}} \sqrt{u_{1}^{2}+u_{2}^{2}} \mathrm{~d} t
$$

This length represents the cost, i.e., the quantity that has to be minimized in our problem. This cost is time-reparametrization invariant. Thus, with such a cost one can always reparametrize the time of the optimal solution in order to obtain controls with slow variation (i.e., with $\dot{u}_{1}$ and $\dot{u}_{2}$ small), that are closer to realistic pulses in practice.

Standard considerations show that it is equivalent to minimize the fluence for fixed transfer time $t_{1}-t_{0}$ :

$$
\mathcal{E}=\int_{t_{0}}^{t_{1}}\left(u_{1}^{2}+u_{2}^{2}\right) d t
$$


instead of the length $\ell(20)$. Indeed a curve minimizes the cost $\mathcal{E}$ among all curves joining the points $q_{0}$ and $q_{1}$ in time $t_{1}-t_{0}$ if:

(a) it minimizes the length $\ell$ among all curves joining $q_{0}$ to $q_{1}$, and

(b) it is a curve parametrized by a multiple of the arclength.

Once we have a curve minimizing $\mathcal{E}$, we can then change the parametrization to have derivatives of controls as small as required.

Remark 7: Notice that, if we do not fix the time, the minimum of the cost $\mathcal{E}$ is zero and it is not attained.

\section{Minimizing the time}

Reparametrizing the geodesics of the control system (19), (20) by the arclength, i.e., setting

$$
\mathrm{d} s=\sqrt{u_{1}^{2}+u_{2}^{2}} \mathrm{~d} t,
$$

we get the control problem:

$$
\dot{\mathbf{x}}=u_{1} F_{1}+u_{2} F_{2}, \quad u_{1}^{2}+u_{2}^{2}=1,
$$

and the question is to minimize the transfer time, from the initial to the terminal condition.

Taking into account the homogeneity of the problem (22), it is clear that it is the same as to minimize the transfer time for the system $\dot{\mathbf{x}}=u_{1} F_{1}+u_{2} F_{2}$, with the constraint

$$
u_{1}^{2}+u_{2}^{2} \leqslant 1 \text {. }
$$

It follows that, for the control system (19), the problem of minimizing the cost (20), using a parametrization with arclength [that is using the cost (21), with $t_{1}-t_{0}$ equal to the total length], is equivalent to minimizing the time under the constraint on the controls $u_{1}^{2}+u_{2}^{2} \leqslant 1$.

Remark 8: Notice that the transformation on the controls necessary to eliminate the drift does not affect the conditions $u_{1}^{2}+u_{2}^{2} \leqslant 1, u_{1}^{2}+u_{2}^{2}=1$.

Remark 9: The problem of minimizing the transfer time for the system (19) under the constraint $u_{1}^{2}+u_{2}^{2} \leqslant 1$, makes sense by itself. However, notice that if we drop the condition $u_{1}^{2}+u_{2}^{2}$ $\leqslant 1$, then by the Hermann-Nagano theorem, the minimizing time between any two initial and terminal conditions is zero.

In a recent article ${ }^{12}$ some related questions in the control of spin systems were discussed. The problem is, however, distinctly different from the ones considered here. The authors also consider a quantum system with a drift:

$$
\dot{\mathbf{x}}=X_{0}(\mathbf{x})+\sum_{i=1}^{p} u_{i} X_{i}(\mathbf{x})
$$

left invariant on some compact group $G$. But, in their case, all vector fields $X_{i}(x)$ belong to $\mathbf{k}$, a Lie subalgebra of the Lie algebra $\mathbf{g}$ of $G$. They consider a Cartan decomposition $\mathbf{g}=\mathbf{k} \oplus \mathbf{p}$, with the standard Cartan's commutation relations, and hence, the Lie algebra generated by the $X_{i}$ 's, $i>1$, is not equal to $\mathbf{g}$ (it is only $\mathbf{k}$ ). Therefore in their case, to move from a point in a coset $K$ - $x_{0}$ to another point in a coset $K \cdot x_{1}$ requires the use of the drift $X_{0}$ and hence requires a bounded speed.

This implies that, in their case, even for unbounded controls there is a minimum time, which is strictly larger than zero (and not attained in general). As mentioned previously, if we relax the constraint $u_{1}^{2}+u_{2}^{2} \leqslant 1$, in our case, the minimum time is zero (also not attained in general). 


\section{Conclusions on the choice of the costs}

There are three costs under consideration:

(a) The length (20). In the two-level case it is the sub-Riemannian length and coincides with the integral of the absolute value of the amplitude. In the three-level case it is a singularRiemannian length.

(b) The fluence (21), for fixed transfer time $t_{1}-t_{0}$.

(c) The time under the constraint $u_{1}^{2}+u_{2}^{2} \leqslant 1$.

Only the first of these costs is parametrization invariant, but all cases (a), (b), (c) lead to the same trajectories in the phase space.

\section{THE TWO-LEVEL SYSTEM}

In this section, we study a two-level quantum system in interaction with a laser for which we control both the amplitude and the phase. The Hamiltonian becomes after elimination of the drift

$$
H=\left(\begin{array}{cc}
0 & u(t) \\
u^{*}(t) & 0
\end{array}\right) .
$$

Our aim is to transfer all the population from level $E_{1}$ to level $E_{2}$ minimizing (here we set $t_{0}$ $=0)$

$$
\int_{0}^{t_{1}}|\Omega(t)| \mathrm{d} t=\int_{0}^{t_{1}}|u(t)| \mathrm{d} t .
$$

Writing $\psi(t)=c_{1}(t) \varphi_{1}+c_{2}(t) \varphi_{2}$, where $\varphi_{1}=(1,0), \varphi_{2}=(0,1)$, we start from any point satisfying $\left|c_{1}(0)\right|^{2}=1$, and our target is defined by $\left|c_{2}\left(t_{1}\right)\right|^{2}=1$.

Remark 10: This new Hamiltonian clearly gives rise to a driftless (or "distributional") control system, while the original Hamiltonian (2) had a drift term. Notice that since we have assumed $\Omega \in \mathbb{C}$, this simplification works without any additional hypothesis on $\Omega$. The fact that the optimal strategy has the laser in resonance [i.e., $\Omega(t)=b(t) e^{i[\omega t+\alpha]}, \omega=E_{2}-E_{1}, \alpha \in \mathbb{R}, b($.$) real func-$ tion] will be obtained as a consequence.

The Schrödinger equation corresponding to the Hamiltonian given by formula (23) is equivalent to the system of ordinary differential equations (ODE) for the $c_{i}$ 's:

$$
\left\{\begin{array}{c}
\dot{c}_{1}=-i u(t) c_{2}, \\
\dot{c}_{2}=-i u^{*}(t) c_{1} .
\end{array}\right.
$$

Setting $c_{1}=x_{1}+i x_{2}, c_{2}=x_{3}+i x_{4}, u=u_{1}+i u_{2}$, Eq. (25) becomes

$$
\dot{\mathbf{x}}=u_{1} F_{1}+u_{2} F_{2},
$$

where

$$
\mathbf{x}=\left(\begin{array}{c}
x_{1} \\
x_{2} \\
x_{3} \\
x_{4}
\end{array}\right), \quad F_{1}=\left(\begin{array}{c}
x_{4} \\
-x_{3} \\
x_{2} \\
-x_{1}
\end{array}\right), \quad F_{2}=\left(\begin{array}{c}
x_{3} \\
x_{4} \\
-x_{1} \\
-x_{2}
\end{array}\right)
$$

and the functional (24) to be minimized is now

$$
\int_{0}^{t_{1}} \sqrt{u_{1}^{2}+u_{2}^{2}} \mathrm{~d} t
$$


In these new variables the condition $\left|c_{1}(t)\right|^{2}+\left|c_{2}(t)\right|^{2}=1$ is $\Sigma_{i=1}^{4} x_{i}^{2}(t)=1$, so in fact $\mathbf{x} \in S^{3}$. The initial condition is now one point on the circle $S_{i n}^{1}:=\left\{\mathbf{x} \in S^{3}: x_{1}^{2}+x_{2}^{2}=1\right\}$ and the target is the circle $S_{\text {fin }}^{1}:=\left\{\mathbf{x} \in S^{3}: x_{3}^{2}+x_{4}^{2}=1\right\}$. These targets are preserved by the transformations eliminating the drift. The problem of minimizing (27) is a classical sub-Riemannian problem on $S^{3}$, which is contact, as we shall see immediately.

The Lie algebra of the distribution. Let us compute the Lie algebra of the distribution. By setting $F_{3}=\left[F_{1}, F_{2}\right]$, we have

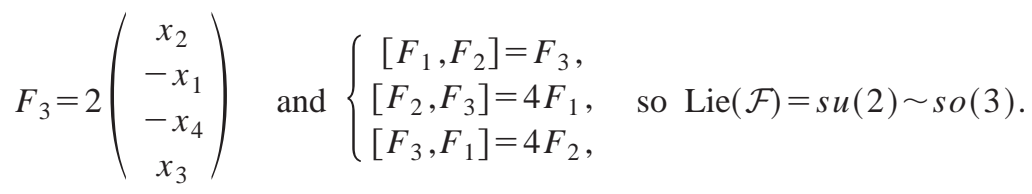

\section{A. Controllability and minimizers}

Let $\mathcal{F}:=\left\{F_{1}, F_{2}\right\}$. Since $\mathcal{F}$ is an analytic family of vector fields on an analytic manifold, we can use the Hermann-Nagano theorem (see Sec. I). In this case, $\operatorname{Lie}_{\mathbf{x}_{0}}(\mathcal{F})$ is the vector space having $F_{1}(x), F_{2}(x)$, and $F_{3}(x)$ as base vectors. The Hermann-Nagano theorem says that the orbit is an analytic submanifold of $S^{3}$ of dimension given by $\operatorname{Lie}_{\mathbf{x}}(\mathcal{F})$, where $\mathbf{x}$ is any point of the orbit. Let $n(\mathbf{x}), \mathbf{x} \in S^{3}$ be the rank of the distribution. We have:

$$
n(\mathbf{x}):=\operatorname{rank}_{\mathbf{x}}\left(F_{1}, F_{2}, F_{3}\right)=\operatorname{rank}_{\mathbf{x}}\left(\begin{array}{ccc}
x_{4} & x_{3} & 2 x_{2} \\
-x_{3} & x_{4} & -2 x_{1} \\
x_{2} & -x_{1} & -2 x_{4} \\
-x_{1} & -x_{2} & 2 x_{3}
\end{array}\right)=3 .
$$

It follows:

Proposition 1: The control system (26) is completely controllable.

Remark 11: This controllability property, using only the first bracket, is equivalent, in the three-dimensional case, to the fact that the distribution is contact.

Remark 12: The sub-Riemannian problem is not generic at all: first as we shall see, it is isoperimetric (it has a symmetry). Second, even among isoperimetric sub-Riemannian problems, it is non generic in the sense that the main basic sub-Riemannian invariant vanishes (see Refs. 19, 23, and 24).

Remark 13: The control system (26) is invariant under the following transformation:

$$
\left(\begin{array}{c}
x_{1} \\
x_{2} \\
x_{3} \\
x_{4}
\end{array}\right) \rightarrow\left(\begin{array}{cccc}
\cos (\alpha) & \sin (\alpha) & 0 & 0 \\
-\sin (\alpha) & \cos (\alpha) & 0 & 0 \\
0 & 0 & \cos (\alpha) & \sin (\alpha) \\
0 & 0 & -\sin (\alpha) & \cos (\alpha)
\end{array}\right)\left(\begin{array}{c}
x_{1} \\
x_{2} \\
x_{3} \\
x_{4}
\end{array}\right)
$$

This means that all the initial conditions $\mathbf{x}_{0} \in S_{\text {in }}$ are equivalent. Notice that transformation (29) is generated by the Lie bracket $\left[F_{1}, F_{2}\right]=F_{3}$.

Minimizing geodesics. We will be able to find optimal trajectories joining our boundary conditions without making any computation. In fact $F_{1}$ and $F_{2}$ are two orthogonal vectors for the standard metric of $S^{3}$ :

$$
F_{1} \cdot F_{2}=\sum_{i=1}^{4}\left(F_{1}\right)_{i}\left(F_{2}\right)_{i}=0
$$

Hence, the length of an admissible curve is just its standard Riemannian length on $S^{3}$. Therefore, if we find an admissible trajectory going from state 1 to state 2 , which is a minimizing geodesic 


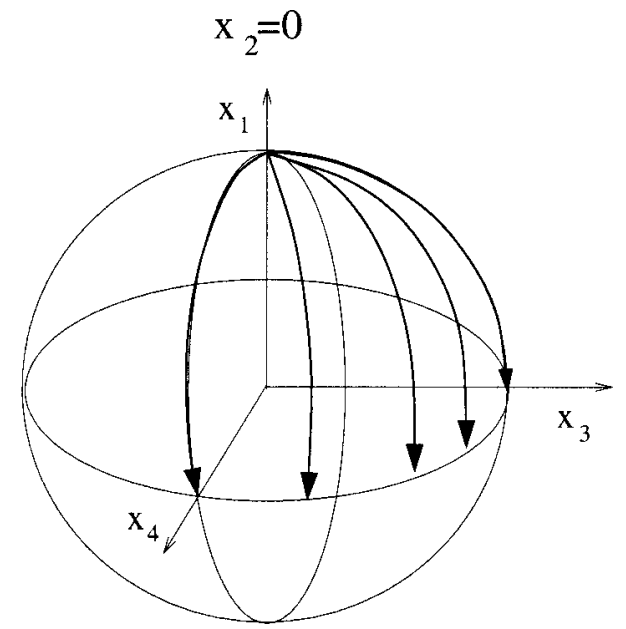

FIG. 1. The sphere $S^{3}$ and the geodesics from $x_{1}=1$ in the space $x_{2}=0$.

for the Riemannian metric on $S^{3}$, then it is also a minimizer for our sub-Riemannian problem. Now, each integral curve of the vector field $b\left(\cos (\alpha) F_{1}+\sin (\alpha) F_{2}\right)(b \in \mathbb{R}, \alpha \in[0,2 \pi])$ are such admissible trajectories. It follows that:

Theorem 1: Every constant control of the form:

$$
\left\{\begin{array}{l}
u_{1}=b \cos (\alpha) \\
u_{2}=b \sin (\alpha)
\end{array}, \quad b \in \mathbb{R}, \quad \alpha \in[0,2 \pi]\right.
$$

is optimal and the target is reached at time $t_{1}=(\pi / 2)(1 / b)$. Moreover if $\mathbf{x}_{0} \in S_{\text {in }}$ is defined by $x_{1}=\cos (\beta), x_{2}=\sin (\beta)$, then the equations of the geodesics corresponding to the controls (30) are:

$$
\left\{\begin{array}{l}
x_{1}=\cos (\beta) \cos (b t), \\
x_{2}=\sin (\beta) \cos (b t), \\
x_{3}=-\sin (\alpha-\beta) \sin (b t), \\
x_{4}=-\cos (\alpha-\beta) \sin (b t) .
\end{array}\right.
$$

The second part of Theorem 1 is easily checked.

Now since the functional (27) is invariant under time reparametrizations, one can take $b$ to be a function of the time so that every optimal control has the form given by

$$
\Omega(t)=b(t) e^{i\left[\left(E_{2}-E_{1}\right) t+\alpha\right]},
$$

where $\alpha$ is an arbitrary constant, $b():. \mathbb{R} \rightarrow \mathbb{R}^{+}$is a real function with compact support $\left[0, t_{1}\right]$ and satisfying $\int_{0}^{t_{1}} b(t)=\pi / 2$. In Fig. 1 are shown the geodesics issued from the point $x_{1}=1$ in the space $x_{2}=0$.

\section{B. Another interpretation: The Hopf fibration and the Isoarea problem}

The Hopf fibration (see, e.g., Ref. 18), $\pi: S^{3} \rightarrow S^{2}$ is defined by the following map. Let $S^{3}$ be described by the variables $\left(x_{1}, x_{2}, x_{3}, x_{4}\right), \Sigma x_{i}^{2}=1$ and $S^{2}$ by the variables $\left(z_{1}, z_{2}, z_{3}\right), \Sigma z_{i}^{2}$ $=1 / 4$. We have:

$$
\left(z_{1}, z_{2}, z_{3}\right)=\pi\left(x_{1}, x_{2}, x_{3}, x_{4}\right):=\left(\frac{1}{2}\left(x_{1}^{2}+x_{2}^{2}-x_{3}^{2}-x_{4}^{2}\right), x_{1} x_{3}-x_{2} x_{4}, x_{2} x_{3}+x_{1} x_{4}\right) .
$$




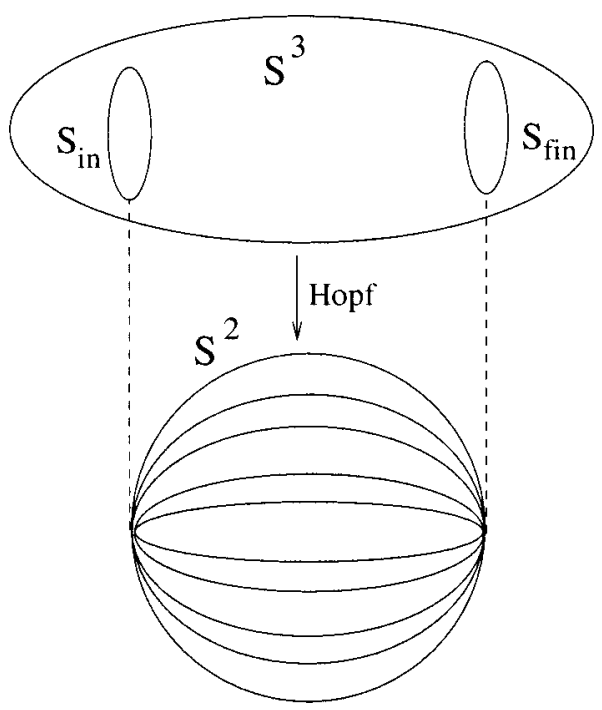

FIG. 2. Schematic representation of the Hopf fibration $\pi: S^{3} \rightarrow S^{2}$. The geodesics given by Theorem 1 are also drawn.

The Hopf fibration gives to $S^{3}$ the structure of a principal bundle with base $S^{2}$ and fiber $S^{1}$ $\sim U(1)$. The following proposition (that one easily checks) shows why the Hopf fibration is connected to our problem:

Proposition 2: Let $F_{3}:=\left[F_{1}, F_{2}\right]$ [see formula (28)], and $\pi$ the Hopf fibration defined above. Then $F_{3} \in \operatorname{Ker}(d \pi)$.

Notice that $F_{3}$ is the generator of the symmetry that "transports" along $S_{\text {in }}$ and $S_{\text {fin }}$ (cf. Remark 13) that means the following. If $\mathbf{x}_{0} \in S_{\text {in }}$ (respectively, $\mathbf{x}_{0} \in S_{\text {fin }}$ ) then the orbit $\mathcal{O}\left(x_{0}\right)$ of $F_{3}$ coincides with $S_{\text {in }}$ (respectively, $S_{\text {fin }}$ ). From Proposition 2 it follows that $S_{\text {in }}$ and $S_{\text {fin }}$ shrink into two points through $\pi$. In particular these points are, respectively, the opposite points $\left(z_{1}, z_{2}, z_{3}\right)$ $=\left( \pm \frac{1}{2}, 0,0\right)$. The situation is illustrated by Fig. 2 . Notice that we have a one parameter family of geodesics connecting $S_{\text {in }}$ and $S_{\text {fin }}$, since their images under $\pi$ are opposite points on $S^{2}$.

In fact, the sub-Riemannian problem we have, is as follows: The distribution (transversal to the fibers of the Hopf fibration) defines a connection over this (circle) principal bundle. It is easily seen that the curvature form of this connection is just the pull back (by the bundle projection) of the volume form of the Euclidean metric on $S^{2}$.

As a consequence (see Ref. 19), our sub-Riemannian problem corresponds to the "isoarea problem" on the Riemannian sphere $S^{2}$ : given two points (antipodal on $S^{2}$ in our case), and any fixed curve $x($.$) joining these two points, find another curve y($.$) , joining also the two points, such$ that the length of $y($.$) is minimal, and the area encircled by the curves x(),. y($.$) has a given value.$

Corollary: There is no other minimizing curve [than the circle (31)] joining $S_{\text {in }}$ and $S_{\text {fin }}$.

Proof: Assume that $\gamma:\left[0, t_{1}\right] \rightarrow S^{3}$ is such a minimizing curve. Then it has length $\pi / 2$, and its projection on $S^{2}$ is one of the circles in Fig. 2. Now each of these circles is lifted in a unique way, via the connection, in one of the curves (31), once the initial point is chosen in $S_{\text {in }}$. Hence $\gamma$ is one of the circles (31).

Our (very special) solutions exhibited previously are in fact geodesics of the Euclidean metric on $S^{2}$ : they correspond to the choice of geodesics for the curve $x($.$) , and to the zero value of the$ area.

\section{THE THREE-LEVEL RESONANT PROBLEM}

Statement of the Problem. In this section, we study a three-level quantum system, with only neighboring levels coupled, controlled by two laser pulses in resonance, i.e., with frequencies $\omega_{1}=E_{2}-E_{1}, \omega_{2}=E_{3}-E_{2} ; E_{1}, E_{2}, E_{3}$ being the three energy levels. 
The aim is to transfer all the population from the state with energy $E_{1}$ to the state with energy $E_{3}$ minimizing (again we set $t_{0}=0$ )

$$
\int_{0}^{t_{1}} \sqrt{u_{1}^{2}+u_{2}^{2}} \mathrm{~d} t
$$

Writing $\psi(t)=c_{1}(t) \varphi_{1}+c_{2}(t) \varphi_{2}+c_{3}(t) \varphi_{3}$, where $\varphi_{1}=(1,0,0), \varphi_{2}=(0,1,0), \varphi_{3}=(0,0,1)$, we start from one point satisfying $\left|c_{1}(0)\right|^{2}=1$, and our target is defined by $\left|c_{3}\left(t_{1}\right)\right|^{2}=1$.

Remark 14: As in Sec. III, this new Hamiltonian gives rise to a driftless autonomous control system, while the control system corresponding to the old Hamiltonian (6) was time dependent and with drift. But in this case (i.e., with real controls), to obtain this strong simplification it is essential to use lasers in resonance.

The Control System. The Schrödinger equation corresponding to the Hamiltonian given by formula (15) is equivalent to the system of ODE for the $c_{i}$ :

$$
\left\{\begin{array}{l}
\dot{c}_{1}=-i u_{1}(t) c_{2}, \\
\dot{c}_{2}=-i\left(u_{1}(t) c_{1}+u_{2}(t) c_{3}\right), \\
\dot{c}_{3}=-i u_{2}(t) c_{2} .
\end{array}\right.
$$

Setting $c_{1}=x_{1}+i x_{2}, c_{2}=x_{4}-i x_{3}, c_{3}=x_{5}+i x_{6}$, Eq. (34) becomes

$$
\dot{\mathbf{x}}=u_{1} F_{1}+u_{2} F_{2}
$$

where

$$
x=\left(\begin{array}{c}
x_{1} \\
x_{2} \\
x_{3} \\
x_{4} \\
x_{5} \\
x_{6}
\end{array}\right), \quad F_{1}=\left(\begin{array}{c}
-x_{3} \\
-x_{4} \\
x_{1} \\
x_{2} \\
0 \\
0
\end{array}\right), \quad F_{2}=\left(\begin{array}{c}
0 \\
0 \\
x_{5} \\
x_{6} \\
-x_{3} \\
-x_{4}
\end{array}\right) .
$$

Here, the notations are such that the real parts (respectively, imaginary parts) of $c_{1}, c_{2}, c_{3}$ are $x_{1}, x_{4}, x_{5}$ (respectively $\left.x_{2},-x_{3}, x_{6}\right)$. This convention will be very convenient later on.

In these new variables the condition $\left|c_{1}(t)\right|^{2}+\left|c_{2}(t)\right|^{2}+\left|c_{3}(t)\right|^{2}=1$ is $\sum_{i=1}^{6} x_{i}^{2}(t)=1$, so in fact $\mathbf{x} \in S^{5}$. The initial condition is now one point on the circle $S_{\text {in }}^{1}:=\left\{\mathbf{x} \in S^{5}: x_{1}^{2}+x_{2}^{2}=1\right\}$ and the target is $S_{\text {fin }}^{1}:=\left\{\mathbf{x} \in S^{5}: x_{5}^{2}+x_{6}^{2}=1\right\}$. With the choice (33) of the functional to be minimized, our problem looks like a classical sub-Riemannian problem on $S^{5}$, but, as we shall see, it is very degenerate.

The Lie algebra of the distribution. Let us compute the Lie algebra of the distribution. By setting $F_{3}=\left[F_{1}, F_{2}\right]$, we have

$$
F_{3}=\left(\begin{array}{c}
x_{5} \\
x_{6} \\
0 \\
0 \\
-x_{1} \\
-x_{2}
\end{array}\right), \quad\left\{\begin{array}{l}
{\left[F_{1}, F_{2}\right]=F_{3},} \\
{\left[F_{2}, F_{3}\right]=F_{1},} \\
{\left[F_{3}, F_{1}\right]=F_{2},}
\end{array}\right.
$$

so $\operatorname{Lie}(\mathcal{F})=\operatorname{su}(2) \sim \operatorname{so}(3)$. 


\section{A. General properties of the orbit}

Let $n(\mathbf{x}), \mathbf{x} \in S^{5}$ be the rank of the distribution. We have

$$
n(\mathbf{x}):=\operatorname{rank}_{\mathbf{x}}\left(F_{1}, F_{2}, F_{3}\right)=\operatorname{rank}_{\mathbf{x}}\left(\begin{array}{ccc}
-x_{3} & 0 & x_{5} \\
-x_{4} & 0 & x_{6} \\
x_{1} & x_{5} & 0 \\
x_{2} & x_{6} & 0 \\
0 & -x_{3} & -x_{1} \\
0 & -x_{4} & -x_{2}
\end{array}\right)= \begin{cases}2 & \text { if } \mathbf{x} \in Q \\
3 & \text { if } \mathbf{x} \in S^{5} \backslash Q,\end{cases}
$$

where $Q$ is the subset of $S^{5}$ defined by

$$
\begin{aligned}
& x_{3} x_{6}-x_{4} x_{5}=0, \\
& x_{1} x_{6}-x_{2} x_{5}=0, \\
& x_{3} x_{2}-x_{4} x_{1}=0 .
\end{aligned}
$$

Notice that if $x_{1}, x_{3}, x_{5}$ are all different from zero, Eqs. (37), (38), and (39) are equivalent to

$$
\frac{x_{2}}{x_{1}}=\frac{x_{4}}{x_{3}}=\frac{x_{6}}{x_{5}} .
$$

Now since every initial condition lies in $Q$ (i.e., $S_{\text {in }}^{1} \in Q$ ), from the Hermann-Nagano theorem it follows:

Proposition 3: For each $\mathbf{x}_{0} \in S_{\text {in }}^{1}$, the orbit $\mathcal{O}\left(\mathbf{x}_{0}\right)$ is an analytic two-dimensional submanifold of $S^{5}$.

More precisely defining $\mathbf{x}_{0}(\alpha)$ as the initial condition corresponding to

$$
\left\{\begin{array}{l}
x_{1}(0)=\cos (\alpha), \\
x_{2}(0)=\sin (\alpha),
\end{array}\right.
$$

where $\alpha \in[0,2 \pi[$, we get the following:

Theorem 2: The orbit $O\left(\mathbf{x}_{0}(\alpha)\right), \alpha \in\left[0,2 \pi\left[\right.\right.$ is the two-dimensional sphere of equation $x_{1}^{\prime 2}$ $+x_{3}^{\prime 2}+x_{5}^{\prime 2}=1$ where

$$
\left(\begin{array}{c}
x_{1}^{\prime} \\
x_{2}^{\prime} \\
x_{3}^{\prime} \\
x_{4}^{\prime} \\
x_{5}^{\prime} \\
x_{6}^{\prime}
\end{array}\right)=\left(\begin{array}{cccccc}
\cos (\alpha) & \sin (\alpha) & 0 & 0 & 0 & 0 \\
-\sin (\alpha) & \cos (\alpha) & 0 & 0 & 0 & 0 \\
0 & 0 & \cos (\alpha) & \sin (\alpha) & 0 & 0 \\
0 & 0 & -\sin (\alpha) & \cos (\alpha) & 0 & 0 \\
0 & 0 & 0 & 0 & \cos (\alpha) & \sin (\alpha) \\
0 & 0 & 0 & 0 & -\sin (\alpha) & \cos (\alpha)
\end{array}\right)\left(\begin{array}{c}
x_{1} \\
x_{2} \\
x_{3} \\
x_{4} \\
x_{5} \\
x_{6}
\end{array}\right)
$$

Proof: Assume first $\alpha=0$. This means that the initial condition is defined by $x_{1}(0)=1$ and the variables with the prime coincide with those without the prime.

In Eq. (35) the variables $x_{1}, x_{3}, x_{5}$ are decoupled from the other (in fact, we have a product system, of two subsystems on $\left.\mathbb{R}^{3}\right)$. It follows $\mathcal{O}\left(\mathbf{x}_{0}(\alpha)\right) \subset\left\{\mathbf{x} \in S^{5}: x_{1}^{2}+x_{3}^{2}+x_{5}^{2}=1\right\}$. But all the points of this sphere belong to the orbit since they can be reached by using a control of the form:

$$
\left(u_{1}(t), u_{2}(t)\right)= \begin{cases}(1,0) & \text { for } t \in\left[0, t_{a}\right] \\ (0,1) & \text { for } t \in\left[t_{a}, t_{b}\right]\end{cases}
$$



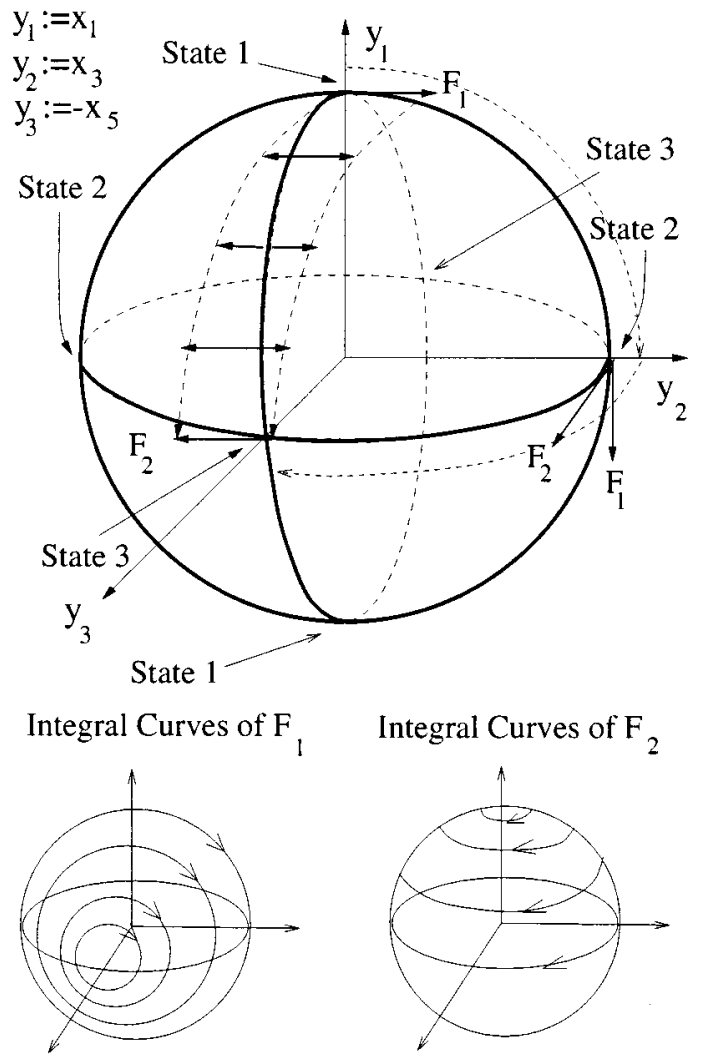

FIG. 3. The vector fields $F_{1}$ and $F_{2}$ on the sphere.

for some $t_{a}, t_{b}>0$. In fact, in the $\left(x_{1}, x_{3}, x_{5}\right)$ space, each integral curve of $F_{1}$ (respectively, $\left.F_{2}\right)$ is a circle that lies in the plane $x_{5}=$ const (respectively, $x_{1}=$ const) and the center of which belongs to the $x_{5}$ (respectively, $x_{1}$ ) axis, see Fig. 3 . At time $t_{a}$ one reaches the point:

$$
\left\{\begin{array}{l}
x_{1}\left(t_{a}\right)=\cos \left(t_{a}\right), \\
x_{3}\left(t_{a}\right)=\sin \left(t_{a}\right), . \\
x_{5}\left(t_{a}\right)=0 .
\end{array}\right.
$$

At time $t_{b}$ one reaches the point:

$$
\left\{\begin{array}{l}
x_{1}\left(t_{b}\right)=\cos \left(t_{a}\right), \\
x_{3}\left(t_{b}\right)=\sin \left(t_{a}\right) \cos \left(t_{b}\right), \\
x_{5}\left(t_{b}\right)=-\sin \left(t_{b}\right) .
\end{array}\right.
$$

So all the points of the sphere $x_{1}^{2}+x_{3}^{2}+x_{5}^{2}=1$ can be reached for suitable $t_{a}$ and $t_{b}$. The Theorem is proved for $\alpha=0$.

Assume now that we start from the point $\mathbf{x}_{0}(\alpha)$ for an arbitrary $\alpha$. Making the change of coordinates given by formula (42), one is back to the situation $\alpha=0$ for the variables with the prime. This concludes the proof.

Notice that, for fixed $\mathbf{x}_{0}(\alpha)$, one can reach only two points of the final target. For instance if $\alpha=0$, i.e., $x_{1}(0)=1$, we can reach $S_{\text {fin }}^{1}$ only in the two points $x_{5}= \pm 1$. The situation is illustrated by Fig. 4.

More precisely as a consequence of the symmetry given by formula (42) we have the following: 


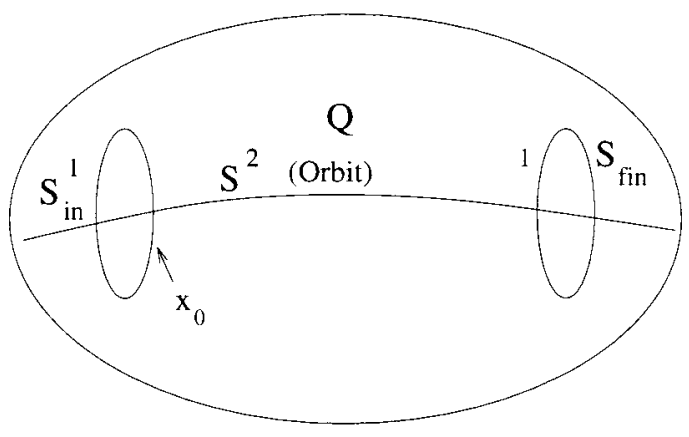

FIG. 4. The foliation of $Q$.

Corollary of Theorem 2: Fix $\alpha \in[0,2 \pi]$, let $\mathbf{x}=\left(x_{1}, \ldots, x_{6}\right) \in \mathcal{O}\left(\mathbf{x}_{0}(\alpha)\right)$, and define $\alpha_{12}(\mathbf{x})$, $\alpha_{34}(\mathbf{x}), \alpha_{56}(\mathbf{x})$ to be the angles (illustrated in Fig. 5) such that

$$
\begin{aligned}
& \left\{\begin{array}{l}
x_{1}=\sqrt{x_{1}^{2}+x_{2}^{2}} \cos \left(\alpha_{12}(\mathbf{x})\right), \\
x_{2}=\sqrt{x_{1}^{2}+x_{2}^{2}} \sin \left(\alpha_{12}(\mathbf{x})\right),
\end{array}\right. \\
& \left\{\begin{array}{l}
x_{3}=\sqrt{x_{3}^{2}+x_{4}^{2}} \cos \left(\alpha_{34}(\mathbf{x})\right), \\
x_{4}=\sqrt{x_{3}^{2}+x_{4}^{2}} \sin \left(\alpha_{34}(\mathbf{x})\right),
\end{array}\right. \\
& \left\{\begin{array}{l}
x_{5}=\sqrt{x_{5}^{2}+x_{6}^{2}} \cos \left(\alpha_{56}(\mathbf{x})\right), \\
x_{6}=\sqrt{x_{5}^{2}+x_{6}^{2}} \sin \left(\alpha_{56}(\mathbf{x})\right) .
\end{array}\right.
\end{aligned}
$$

Then, for each such $\mathbf{x}$, there exist $n_{12}(\mathbf{x}), n_{34}(\mathbf{x}), n_{56}(\mathbf{x}) \in\{0, \pm 1\}$ such that

$$
\begin{aligned}
& \alpha_{12}(\mathbf{x})=\alpha \pm n_{12} \pi \quad \text { if } \quad \alpha_{12} \text { is defined (i.e., } x_{1}, x_{2} \text { not both vanishing), } \\
& \alpha_{34}(\mathbf{x})=\alpha \pm n_{34} \pi \quad \text { if } \quad \alpha_{34} \text { is defined (i.e., } x_{3}, x_{4} \text { not both vanishing), } \\
& \alpha_{56}(\mathbf{x})=\alpha \pm n_{56} \pi \quad \text { if } \quad \alpha_{56} \text { is defined (i.e., } x_{5}, x_{6} \text { not both vanishing). }
\end{aligned}
$$

Moreover at least one of the three angles $\alpha_{12}, \alpha_{34}, \alpha_{56}$ is defined.

The Structure of $Q$. Let us now study the structure of $Q$. From the Hermann-Nagano theorem we have

$$
Q \supseteq \bigcup_{\mathbf{x}_{0} \in S_{\text {in }}^{1}} \mathcal{O}_{\mathbf{x}_{0}}
$$

but in fact this equation holds with equality as a consequence of the fact that relations (37), (38), and (39) defining $Q$ are equivalent to Eqs. (43), (44), (45), that hold on the orbit. More precisely let $\overline{\mathbf{x}}=\left(\bar{x}_{1}, \ldots, \bar{x}_{6}\right)$ be a point of $Q$ and $\bar{\alpha}$ the angle between $\bar{x}_{1}$ and $\bar{x}_{2}$ measured counterclockwise, if defined. If not $\bar{\alpha}$ will be the angle between the variables $\bar{x}_{3}$ and $\bar{x}_{4}$, or between the

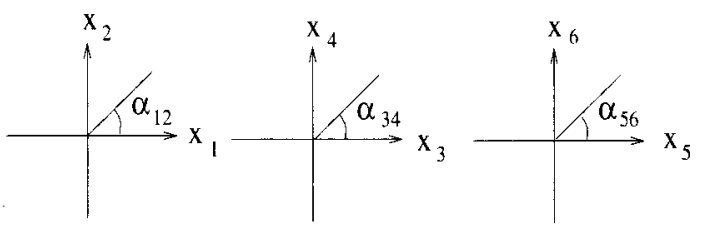

FIG. 5. The definition of the angles $\alpha_{12}(\mathbf{x}), \alpha_{34}(\mathbf{x}), \alpha_{56}(\mathbf{x})$. 


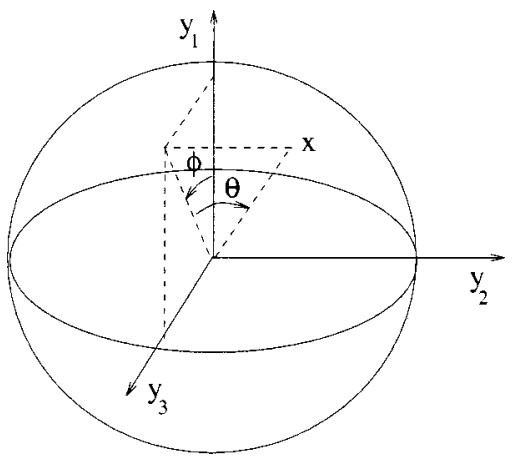

FIG. 6. The angles $\theta$ and $\phi$.

variables $\bar{x}_{5}$ and $\bar{x}_{6}$ (in the spirit of the Corollary of Theorem 2). Because of (40), $\bar{\alpha}$ can be actually defined in this way. One clearly has $\bar{x} \in \mathcal{O}\left(\mathbf{x}_{0}(\bar{\alpha})\right)$. Hence we have proved the following:

Proposition 4: We have $Q=\cup_{\mathbf{x}_{0} \in S_{\text {in }}^{1}} \mathcal{O}_{\mathbf{x}_{0}}$.

Let $\sigma$ be the antipodal involution of $S^{1} \times S^{2}$, that is, $\sigma(\alpha, p)=(\alpha+\pi,-p)$. The involution $\sigma$ has no fixed point, and is orientation reversing. Also, clearly, by Theorem 2, our orbits are $\sigma$ stable. Hence: $Q=\left(S^{1} \times S^{2}\right) / \sim$ where $\mathbf{x} \sim \mathbf{x}^{\prime}$ if $\sigma(x)=\sigma\left(\mathbf{x}^{\prime}\right)$. Therefore, it is not hard to see that:

Theorem 3: $Q$ is the (only) nonorientable sphere-bundle over $S^{1}$.

\section{B. Geodesic equations on the sphere}

Due to the invariance under the transformation (42) all the points of $S_{\text {in }}$ can be considered equivalently. In the following we will study the optimal control problem on the orbit $\mathcal{O}\left(\mathbf{x}_{0}\right)$, where $\mathbf{x}_{0}$ is defined by $x_{1}=1$. Then $\mathcal{O}\left(\mathbf{x}_{0}\right)$ is the sphere of equation $x_{1}^{2}+x_{3}^{2}+x_{5}^{2}=1$. In what follows, we will keep the notation $F_{1}$ and $F_{2}$ for the restrictions of $F_{1}$ and $F_{2}$ to this sphere.

In order to get labels for coordinates corresponding to quantum states, and in order to have $F_{2}$ pointing in the positive direction from the point $x_{3}=1$, we define $y_{1}=x_{1}, y_{2}=x_{3}, y_{3}=-x_{5}$. The control system under consideration is then

$$
\left(\begin{array}{c}
\dot{y}_{1} \\
\dot{y}_{2} \\
\dot{y}_{3}
\end{array}\right)=u_{1} F_{1}+u_{2} F_{2},
$$

where

$$
F_{1}=\left(\begin{array}{c}
-y_{2} \\
y_{1} \\
0
\end{array}\right), \quad F_{2}=\left(\begin{array}{c}
0 \\
-y_{3} \\
y_{2}
\end{array}\right) .
$$

The vector fields are plotted in Fig. 3. The initial condition is the point $y_{1}=1$. The state number 1 (respectively, 2,3) correspond to the points $y_{1}= \pm 1$ (respectively, $y_{2}= \pm 1, y_{3}= \pm 1$ ). State 2 can be reached from state 1 using only $F_{1}$ and state 3 can be reached from state 2 using only $F_{2}$ (dotted lines). However, state 3 cannot be reached from state 1 using a trajectory contained in the circle of equation:

$$
\left.y_{2}=0 \quad \text { (i.e., } y_{1}^{2}+y_{3}^{2}=1\right) \text {, }
$$

since no piece of this circle is an admissible trajectory. This is due to the fact that $F_{1}$ is collinear to $F_{2}$ on the circle (46), and not tangent to this circle.

A new orthogonal frame. Let us describe the orbit in spherical coordinates (see Fig. 6): 


$$
\left\{\begin{array}{l}
y_{1}=\cos (\theta) \cos (\phi), \\
y_{2}=\sin (\theta), \\
y_{3}=\cos (\theta) \sin (\phi) .
\end{array}\right.
$$

We have

$$
\left(\begin{array}{l}
F_{1} \\
F_{2}
\end{array}\right)=R\left(\begin{array}{l}
G_{1} \\
G_{2}
\end{array}\right)
$$

where

$$
R:=\left(\begin{array}{cc}
\cos (\phi) & \sin (\phi) \\
-\sin (\phi) & \cos (\phi)
\end{array}\right), \quad\left\{\begin{array}{l}
G_{1}=\partial_{\theta} \\
G_{2}=\tan (\theta) \partial_{\phi} .
\end{array}\right.
$$

Since $R \in \mathrm{SO}(2)$, the couple $\left(G_{1}, G_{2}\right)$ is a new orthonormal frame for the singular-Riemannian length. For this new frame the control system is then

$$
\left(\begin{array}{c}
\dot{\theta} \\
\dot{\phi}
\end{array}\right)=v_{1} G_{1}+v_{2} G_{2}
$$

and the functional to be minimized is $\int_{0}^{t_{1}} \sqrt{v_{1}^{2}+v_{2}^{2}} \mathrm{~d} t$. The relation between $u_{1}, u_{2}$ and $v_{1}, v_{2}$ is obtained from $u_{1} F_{1}+u_{2} F_{2} \equiv v_{1} G_{1}+v_{2} G_{2}$ :

$$
\left(\begin{array}{l}
u_{1} \\
u_{2}
\end{array}\right)=R\left(\begin{array}{l}
v_{1} \\
v_{2}
\end{array}\right) .
$$

The metric defined by the frame $\left(G_{1}, G_{2}\right)$ is a singular metric. Indeed when $\theta=0$ we have $G_{2}$ $=0$, that is exactly on the circle (46). Notice that the singularity of the metric for $\theta=\pi / 2$ is only due to the choice of the coordinates system.

The Hamiltonian. Let us compute the geodesics using the Maximum Principle. Here, for standard reasons, we use as cost the fluence (21). The final time is fixed by requiring the parametrization by arclength that is $v_{1}^{2}+v_{2}^{2}=1$ (this means to normalize $\mathcal{H}_{M}=1 / 2$, see the Pontryagin Maximum Principle in Sec. I for the definition of $\mathcal{H}_{M}$ ). Notice that from formula (49) we have

$$
v_{1}^{2}+v_{2}^{2}=u_{1}^{2}+u_{2}^{2} .
$$

As explained in Sec. IIC 2 this corresponds to minimizing the time under the constraint $u_{1}^{2}$ $+u_{2}^{2} \leqslant 1$. Let $P=\left(P_{\theta}, P_{\phi}\right) \in T_{\theta, \phi}^{*} M$. By definition the Hamiltonian is

$$
\begin{aligned}
\mathcal{H}\left(\theta, \phi, P_{\theta}, P_{\phi}, v_{1}, v_{2}\right) & =\left\langle P, v_{1} G_{1}+v_{2} G_{2}\right\rangle+p_{0}\left(v_{1}^{2}+v_{2}^{2}\right) \\
& =v_{1} P_{\theta}+v_{2} P_{\phi} \tan (\theta)+p_{0}\left(v_{1}^{2}+v_{2}^{2}\right) .
\end{aligned}
$$

It is easily checked that, as for the Riemannian case, we can always assume $p_{0} \neq 0$ (there are no abnormal extremals) and we can normalize $p_{0}=-\frac{1}{2}$. Extremal controls are computed from the maximum condition:

$$
\frac{\partial \mathcal{H}}{\partial v_{1}}=0, \quad \frac{\partial \mathcal{H}}{\partial v_{2}}=0, \quad \Rightarrow v_{1}=P_{\theta}, \quad v_{2}=P_{\phi} \tan (\theta) .
$$

Hence, we obtain that the extremals are projections on the $(\theta, \phi)$ space of integral curves of the Hamiltonian vector field corresponding to the following Hamiltonian:

$$
\mathcal{H}_{M}=\frac{1}{2}\left(P_{\theta}^{2}+\left(\tan (\theta) P_{\phi}\right)^{2}\right) .
$$

The Hamiltonian equations are 


$$
\begin{gathered}
\dot{\theta}=\frac{\partial \mathcal{H}_{M}}{\partial P_{\theta}}=P_{\theta}, \\
\dot{\phi}=\frac{\partial \mathcal{H}_{M}}{\partial P_{\phi}}=P_{\phi} \tan ^{2}(\theta), \\
\dot{P}_{\theta}=-\frac{\partial \mathcal{H}_{M}}{\partial \theta}=-P_{\phi}^{2} \tan (\theta)\left(1+\tan ^{2}(\theta)\right), \\
\dot{P}_{\phi}=-\frac{\partial \mathcal{H}_{M}}{\partial \phi}=0 .
\end{gathered}
$$

Setting $a:=P_{\phi}$ finally we have

$$
\dot{\theta}=P_{\theta}, \quad \dot{\phi}=a \tan ^{2}(\theta), \quad \dot{P}_{\theta}=-a^{2} \tan (\theta)\left(1+\tan ^{2}(\theta)\right) .
$$

This Hamiltonian system is Liouville integrable since we have two independent and commuting constants of the motion $\mathcal{H}_{M}$ and $P_{\phi}=a$.

\section{Minimizing the length}

Let us find the geodesic without the parametrization, i.e., a relation between $\theta$ and $\phi$. We have

$$
\frac{\mathrm{d} \phi}{\mathrm{d} \theta}=\frac{\dot{\phi}}{\dot{\theta}}=\frac{\dot{\phi}}{P_{\theta}}= \pm \frac{a \tan ^{2}(\theta)}{\sqrt{1-a^{2} \tan ^{2}(\theta)}},
$$

where we have expressed $P_{\theta}$ using relation (52) and normalized $\mathcal{H}_{M}=1 / 2$. So we have the two families of solutions parametrized by the value of $a$ :

$$
\begin{aligned}
\phi_{a}^{ \pm}(\theta) & = \pm \int_{0}^{\theta} \frac{a \tan ^{2}(s)}{\sqrt{1-a^{2} \tan ^{2}(s)}} \mathrm{d} s \\
& = \pm\left[\arctan \left(\frac{a}{\Xi(\theta, a)} \sin (\theta)\right)-\frac{a}{\sqrt{1+a^{2}}} \arctan \left(\frac{\sqrt{1+a^{2}}}{\Xi(\theta, a)} \sin (\theta)\right)\right],
\end{aligned}
$$

where $\Xi(\theta, a):=\sqrt{\frac{1}{2}\left(1-a^{2}+\left(1+a^{2}\right) \cos (2 \theta)\right)}$.

To fix the ideas, let us consider only the family $\phi_{a}^{+}$with $a>0$ and suppose $\theta \geqslant 0$, the other cases being symmetric. Let us call this family $\phi_{a}(\theta)$. Expression (55) defines $\phi_{a}(\theta)$ in the interval $\left[0, \bar{\theta}_{a}\right.$ [ where $\bar{\theta}_{a}$ is the value of $\theta$ at which the denominator of formula (54) vanishes:

$$
\bar{\theta}_{a}:=\arctan \left(\frac{1}{a}\right)
$$

Notice that $\lim _{\theta \rightarrow \bar{\theta}_{a}} \Xi[\theta, a]=0$ so

$$
\lim _{\theta \rightarrow \bar{\theta}_{a}} \phi_{a}(\theta)=\frac{\pi}{2}\left(1-\frac{a}{\sqrt{1+a^{2}}}\right) .
$$

In the following we consider $\phi_{a}(\theta)$ defined in $\left[0, \bar{\theta}_{a}\right]$ where by definition $\phi_{a}\left(\bar{\theta}_{a}\right)$ is the value given by formula (57). Indeed, from the symmetries of the system, we have to consider two branches (illustred in Fig. 7) to describe the whole relation between $\theta$ and $\phi$ :

$$
\phi_{a}^{1}(\theta)=\phi_{a}(\theta),
$$




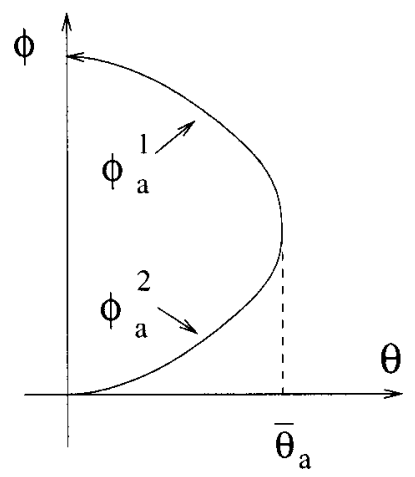

FIG. 7. The geodesic curve as a relation between $\theta$ and $\phi$.

$$
\phi_{a}^{2}(\theta)=2 \phi_{a}\left(\bar{\theta}_{a}\right)-\phi_{a}(\theta) .
$$

The geodesic reaching the target is the one satisfying $\phi_{a}\left(\bar{\theta}_{a}\right)=\pi / 4$. From Eq. (57) we get $a:=1 / \sqrt{3}$.

Remark 15: By considering both signs in formula (55) (or equivalently a positive and negative) and $\theta \in[-\pi, \pi]$ one gets four equivalent optimal trajectories reaching the state 3 (see Fig. 8). Moreover the set of geodesics parametrized by $a$ allows one to easily compute an optimal synthesis for the problem.

A smooth parametrization of the geodesic. To get an explicit expression of the controls (as function of the time) reaching the final point $\theta=0, \phi=\pi / 2$, one should first fix a parametrization $\theta(t)$ in such a way that:

(1) $\theta(0)=\theta\left(t_{1}\right)=0$,

(2) for some $\bar{t} \in] 0, t_{1}[$ it holds:

$$
\left\{\begin{array}{l}
\theta(\bar{t})=\bar{\theta}_{1 / \sqrt{3}}=\pi / 3, \\
\theta(t) \quad \text { is increasing for } t \in[0, \bar{t}], \\
\theta(t) \quad \text { is decreasing for } t \in\left[\bar{t}, t_{1}\right] .
\end{array}\right.
$$

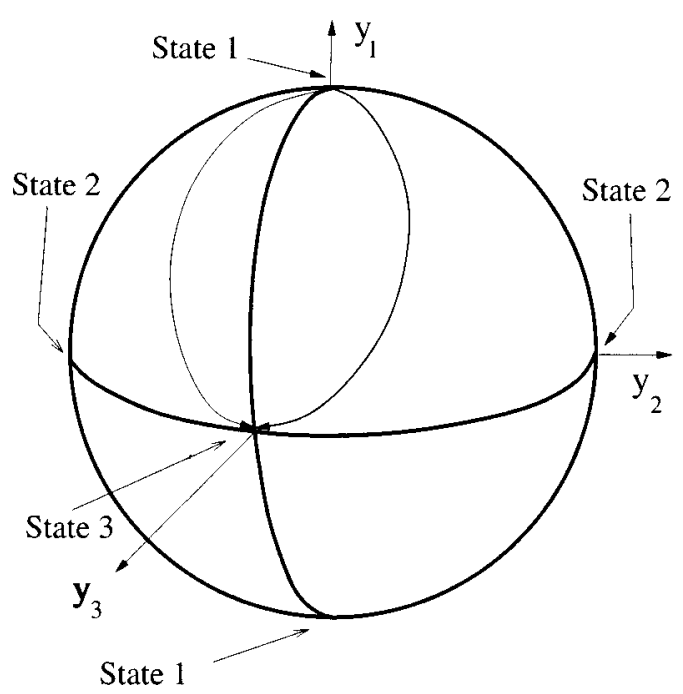

FIG. 8. The four geodesics reaching the state 3. Two are on the other side and reach the point $y_{3}=-1$. 


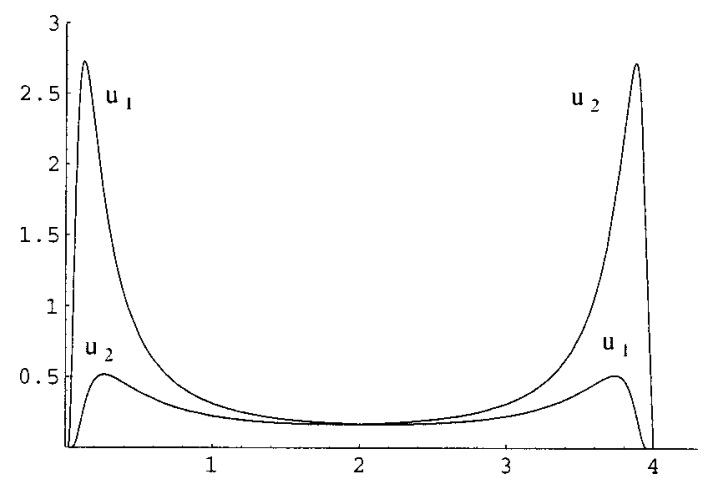

FIG. 9. Example 1: $\mathcal{C}^{\infty}$ optimal controls.

The best choice is of course a sufficiently regular symmetric function. In this case we have $\bar{t}$ $=t_{1} / 2$ and

$$
\phi(t)=\left\{\begin{array}{l}
\phi_{1 / \sqrt{3}}(\theta(t)) \quad \text { if } t \in\left[0, t_{1} / 2[,\right. \\
2 \phi_{1 / \sqrt{3}}\left(\theta\left(t_{1} / 2\right)\right)-\phi_{1 / \sqrt{3}}\left(\theta\left(t_{1}-t\right)\right) \quad \text { if } t \in\left[t_{1} / 2, t_{1}\right] .
\end{array}\right.
$$

The controls $v_{1}(t)$ and $v_{2}(t)$ can be obtained from the relation $\dot{\theta}(t) \partial_{\theta}+\dot{\phi}(t) \partial_{\phi}=v_{1}(t) \partial_{\theta}$ $+v_{2}(t) \tan (\theta(t)) \partial_{\phi}$ from which we have

$$
v_{1}(t)=\dot{\theta}(t), \quad v_{2}(t)=\frac{\dot{\phi}(t)}{\tan (\theta(t))}
$$

Remark 16: Notice that relations (59) coincide with relations (51) only in the case in which the curve $(\theta(t), \phi(t))$ is parametrized with constant velocity.

Finally the amplitudes of the lasers are obtained with formula (49).

Example 1: Consider the $\mathcal{C}^{\infty}$ function:

$$
\theta(t)= \begin{cases}0 & \text { if } t<0 \quad \text { or } t>t_{1} \\ \frac{\pi}{3} e^{1 /\left(t_{1}-t\right)(-t)} & \text { otherwise. }\end{cases}
$$

The corresponding laser amplitudes $u_{1}(t)$ and $u_{2}(t)$ (for $t_{1}=4$ ) are shown in Fig. 9.

Example 2: Consider the function:

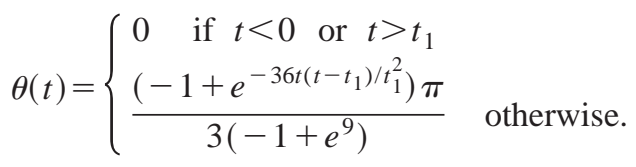

The corresponding laser amplitudes $u_{1}(t)$ and $u_{2}(t)$ (for $\left.t_{1}=4\right)$ are shown in Fig. 10.

\section{Minimizing either the fluence or the time for constrained controls}

To minimize the fluence, one should also get the parametrization from the Hamiltonian equations. From the equation (after fixing $a=1 / \sqrt{3}$ ):

$$
\dot{\theta}=P_{\theta}=\sqrt{1-a^{2} \tan ^{2}(\theta)}
$$

we have 


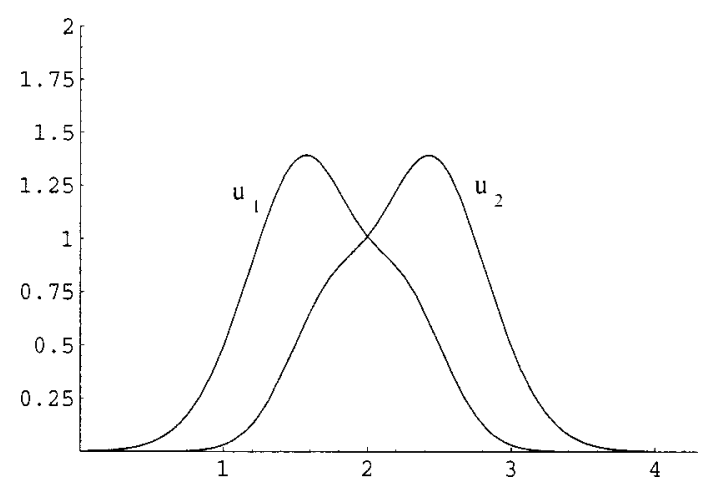

FIG. 10. Example 2: a possible choice of optimal controls.

$$
t(\theta)=\frac{\arctan \left(\frac{2 \sin (\theta)}{\sqrt{1+2 \cos (2 \theta)}}\right) \sqrt{1+2 \cos (2 \theta)} \sec (\theta)}{2 \sqrt{1-\tan (\theta)^{2} / 3}} .
$$

Using the inverse function theorem, this formula permits one to describe $\theta(t)$ in the interval $[0, \bar{t}]$ where

$$
\bar{t}:=\lim _{\theta \rightarrow \bar{\theta}_{a}} t(\theta)=\frac{\sqrt{3}}{4} \pi .
$$

By symmetry this is exactly half of the final time. Finally $\dot{\phi}(t)$ can be obtained integrating the equation $\dot{\phi}(t)=a \tan ^{2}(\theta(t))$. Controls $u_{1}(t)$ and $u_{2}(t)$ can be obtained with formulas (59) and (49) and they are shown in Fig. 11.

Remark 17: Notice that these controls are not smooth at $t=0$ and at the final time $2 \bar{t}$ $=(\sqrt{3} / 2) \pi$.

From this analysis we get the values of the costs for the optimal trajectory:

Theorem 4: For the three costs described in Sec. II C, the following relations hold.

(a) Length:

$$
\operatorname{Min}\left(\int_{0}^{t_{1}} \sqrt{u_{1}^{2}+u_{2}^{2}} \mathrm{~d} t\right)=\frac{\sqrt{3}}{2} \pi
$$

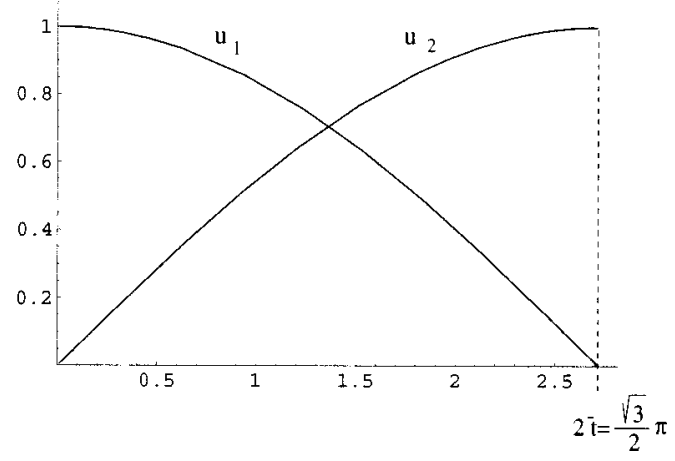

FIG. 11. Optimal controls minimizing fluence or time. 


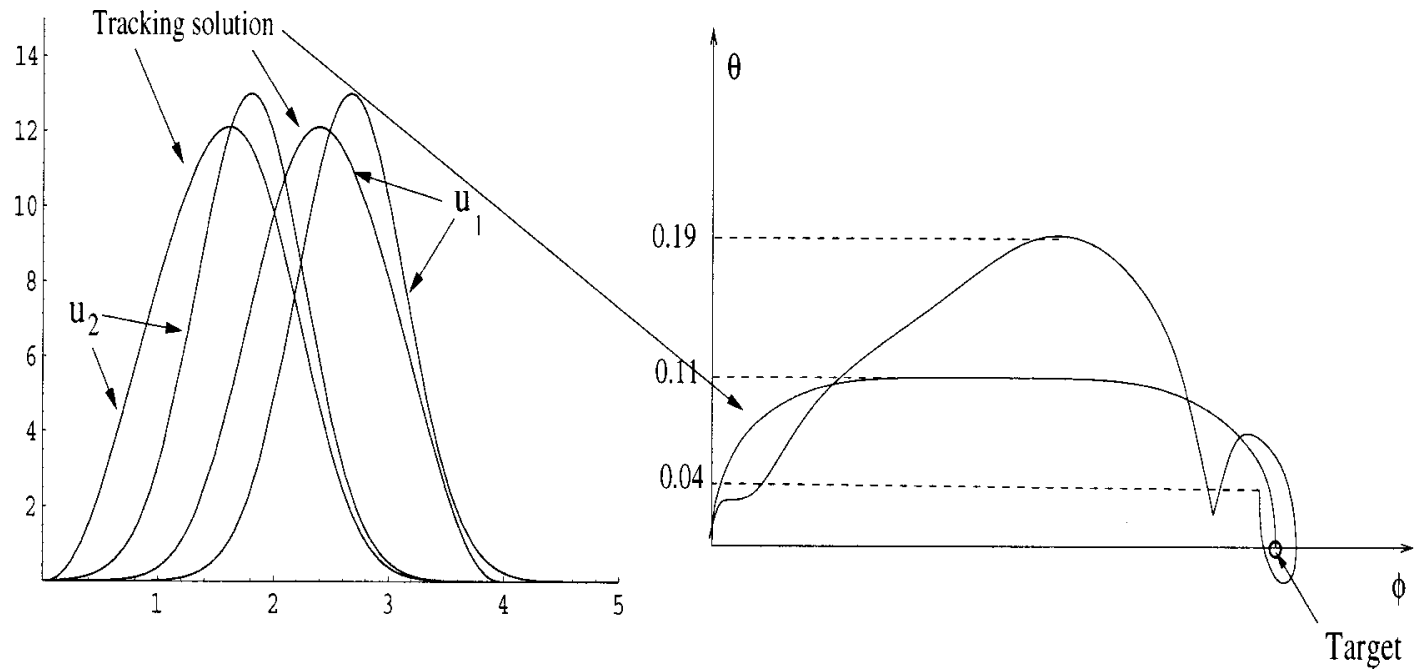

FIG. 12. Usual-adiabatic and tracking solutions.

(b) Fluence, with fixed transfer time T:

$$
\operatorname{Min}\left(\int_{0}^{T}\left(u_{1}^{2}+u_{2}^{2}\right) \mathrm{d} t\right)=\frac{3}{4} \pi^{2} \frac{1}{T} .
$$

(c) Time under the constraint $u_{1}^{2}+u_{2}^{2} \leqslant 1$,

$$
T_{\min }=\frac{\sqrt{3}}{2} \pi .
$$

\section{THE TRACKING COUNTERINTUITIVE SOLUTIONS}

For practical applications, trajectories joining state 1 to state 3, that take as small as possible the population of state 2 [i.e., with $\theta(t)$ closed to zero], are also interesting. We recall that the nonadmissible trajectory is contained in the circle (46). In this section we study a trajectory in which $\phi(t)$ is monotonously increasing between 0 and $\pi / 2$, and

$$
\theta(t) \leqslant \varepsilon, \quad \text { for every } t \in\left[0, t_{1}\right],
$$

for some small $\varepsilon>0$ fixed. This means that the population in state $2\left[0, t_{1}\right]$ is always small, less or equal than $\sin ^{2}(\varepsilon)=\varepsilon^{2}+O\left(\varepsilon^{4}\right)$ [see formula (47)].

It is well known ${ }^{1,25}$ that the nonadmissible trajectory can be approximated using controls $u_{1}(t)$ and $u_{2}(t)$ in the so-called "counterintuitive" sequence: (see Fig. 12 for such a sequence). In the following we show how to build a trajectory satisfying condition (64) and connecting exactly the points $P_{1}$ defined by $(\phi, \theta)=(0,0)$ and $P_{2}$ defined by $(\phi, \theta)=(\pi / 2,0)$.

The idea is to track a trajectory connecting these two points taking care of the constraints that we have on the derivatives at $P_{1}$ and $P_{2}$. More precisely we should find a function $\theta(\phi)$ such that the following holds. Let $\phi^{1}(\theta)$ (respectively, $\phi^{2}(\theta)$ ) be the inverse function of $\theta(\phi)$ in a neighborhood of $P_{1}$ (respectively, $P_{2}$ ). Since $F_{1}$ and $F_{2}$ vanish, respectively, at points $P_{1}$ and $P_{2}$, we must have

$$
\left.\frac{\mathrm{d} \phi^{1}}{\mathrm{~d} \theta}\right|_{\theta=0}=0,
$$




$$
\left.\frac{\mathrm{d} \phi^{2}}{\mathrm{~d} \theta}\right|_{\theta=0}=0
$$

A possible choice is the symmetric function is

$$
\theta(\phi)=\varepsilon \frac{4}{\pi} \sqrt{\phi\left(\frac{\pi}{2}-\phi\right)},
$$

which reaches the value $\varepsilon$ only at the point $\phi=\pi / 4$, as shown in Fig. 12 .

Now we have to choose a parametrization $(\theta(t), \phi(t))$. To have continuous controls satisfying $u_{1}(0)=0, u_{2}(0)=0, u_{1}\left(t_{1}\right)=0, u_{2}\left(t_{1}\right)=0$ we must have

$$
\begin{aligned}
& \left.\dot{\theta}^{1}(t)\right|_{0}=0, \\
& \left.\dot{\theta}^{2}(t)\right|_{t_{1}}=0 .
\end{aligned}
$$

From Eqs. (65), (66) we get

$$
\begin{gathered}
\left.\ddot{\phi}^{1}(t)\right|_{0}=0, \\
\left.\ddot{\phi}^{2}(t)\right|_{t_{1}}=0 .
\end{gathered}
$$

To get controls having zero derivative at the initial and final points, we can use the following function:

$$
\phi(t)=\frac{6006 \pi}{t_{1}^{13}}\left(\frac{t^{13}}{13}-\frac{t^{12} t_{1}}{2}+\frac{15 t^{11} t_{1}^{2}}{11}-2 t^{10} t_{1}^{3}+\frac{5 t^{9} t_{1}^{4}}{3}-\frac{3 t^{8} t_{1}^{5}}{4}+\frac{t^{7} t_{1}^{6}}{7}\right)
$$

Notice that this function satisfies $\phi^{1}(\bar{t})=\phi^{2}(\bar{t})=\phi^{3}(\bar{t})=\phi^{4}(\bar{t})=\phi^{5}(\bar{t})=\phi^{6}(\bar{t})=0$ for $\bar{t}$ $=0, t_{1}$. The corresponding controls are computed with relations (59) and (49).

Remark 18: Let us use a parametrization $\phi(t)$ with $\left.\left.\phi^{(i)}(t)\right|_{t=0}=0,\left.\phi^{(i)}(t)\right|_{t=t_{1}}=0\right), \quad i$ $=1, \ldots, n, n \geqslant 2$, then the following holds:

(1) if $n=2$ then $u_{1}(0)=u_{2}(0)=0, \lim _{t \rightarrow t_{1}} \dot{u}_{1}(t)=-\infty, \lim _{t \rightarrow 0} \dot{u}_{2}(t)=\infty$,

(2) if $n=3$ then $u_{1}(0)=u_{2}(0)=0,0>\dot{u}_{1}\left(t_{1}\right)>-\infty, 0<\dot{u}_{2}(0)<\infty$,

(3) if $n \geqslant 4$ then $u_{1}(0)=u_{2}(0)=0$,

$u_{1}^{(i)}(0)=u_{2}^{(i)}=0$ for $i=1, \ldots, n-3$. So if $\phi$ is $\mathcal{C}^{n}$, in $]-\delta, t_{1}+\delta[(\delta>0, \phi(t)=0$ in ]$-\delta, 0] \cup\left[t_{1}, t_{1}+\delta\left[\right.\right.$ ) then $u_{1}$ and $u_{2}$ are $\mathcal{C}^{n-3}$ in $]-\delta, t_{1}+\delta[$.

Notice that for very small values of $\varepsilon$ one gets very big values of the controls. We would like to stress the fact that the trajectory obtained with this tracking reaches exactly the final target for any fixed value of $\varepsilon$. While in the "counterintuitive" strategies used in literature, if $\varepsilon_{c}$ is the maximum value reached by $\theta$, then the final target is reached with an error smaller than $\varepsilon_{c}$ but different from zero.

In the last picture the tracking solution corresponding to the expression of (68), for $\varepsilon$ $=2 / 19$, and $t_{1}=4$ and a typical strategy used in literature:

$$
\left\{\begin{array}{l}
u_{1}(t)=\frac{-5}{e^{16}}+\frac{13}{e^{(-4+1.5 t)^{2}}}, \\
u_{2}(t)=\frac{-5}{e^{9}}+\frac{13}{e^{(-2.7+1.5 t)^{2}}}
\end{array}\right.
$$


are compared. Notice that the pulses have similar area. Moreover, notice that the trajectory corresponding to the controls (69) (obtained integrating numerically the Schrödinger equation) reaches negative values of $\theta$.

\section{CONCLUSION}

In summary, we have shown how optimal controls for two- and three-level models can be constructed on the basis of geometric arguments. For the two-level modes we recover the wellknown " $\pi$-pulse" strategy, and obtain the corresponding generalization for the resonant threelevel model. The optimal trajectories appear as geodesics (Riemannian or singular-Riemannian) on two-dimensional spheres. Furthermore, besides the optimal control strategies, the standard tracking technique from geometric control theory allows us to analyze a method of adiabatic control used in recent experiments. It leads to an improvement that allows one to reach the target state precisely. The extension of these results to the treatment of more general $\mathrm{N}$-level systems will be presented in forthcoming work.

\section{ACKNOWLEDGMENTS}

This research has been supported by a Marie Curie Fellowship of the European Community. Program "Improving Human Research Potential and the Socio-economic Knowledge Base," Contract No.: HPMF-CT-2001-01479. Moreover we acknowledge support from the Conseil Régional de Bourgogne.

The authors are grateful to Velimir Jurdjevic, Richard Montgomery, and Luisa Paoluzzi for helpful discussions.

\footnotetext{
${ }^{1}$ N. V. Vitanov, T. Halfmann, B. W. Shore, and K. Bergmann, Annu. Rev. Phys. Chem. 52, 763 (2001).

${ }^{2}$ K. Drese and M. Holthaus, Eur. Phys. J. D 5, 119 (1999).

${ }^{3}$ S. Guérin, L. P. Yatsenko, and H. R. Jauslin, Phys. Rev. A 63, 031403(R) (2001).

${ }^{4}$ L. P. Yatsenko, S. Guérin, and H. R. Jauslin, "Topology of adiabatic passage," in LANL e-print quant-ph/0107065, http://xxx.lanl.gov.

${ }^{5}$ M. V. Korolkov, J. Manz, and G. K. Paramonov, Adv. Chem. Phys. 101, 327 (1997).

${ }^{6}$ M. Holthaus and B. Just, Phys. Rev. A 49, 1950 (1994).

${ }^{7}$ M. A. Daleh, A. M. Peirce, and H. Rabitz, Phys. Rev. A 37, 4950 (1988).

${ }^{8}$ A. Bartana, R. Kosloff, and D. J. Tannor, Chem. Phys. 267, 95 (2001).

${ }^{9}$ Y. B. Band and O. Magnes, J. Chem. Phys. 101, 7528 (1994).

${ }^{10}$ V. S. Malinovsky and D. J. Tannor, Phys. Rev. A 56, 4929 (1997).

${ }^{11}$ S. Guérin, S. Thomas, and H. R. Jauslin, Phys. Rev. A 65, 023409 (2002).

${ }^{12}$ N. Khaneja, R. Brockett, and S. J. Glaser, Phys. Rev. A 63, 032308 (2001).

${ }^{13}$ A. Isidori, Nonlinear Control Systems (Springer Verlag, New York, 1995).

${ }^{14}$ B. W. Shore, The Theory of Coherent Atomic Excitation (Wiley, New York, 1990), Vol. 1, pp. 235 and 304

${ }^{15}$ S. Guérin, Phys. Rev. A 56, 1458 (1997).

${ }^{16}$ A. Bellaiche, "The tangent space in sub-Riemannian geometry," Sub-Riemannian Geometry (Birkhuser, Basel, 1996), pp. $1-78$.

${ }^{17}$ M. Gromov, "Carnot-Carathodory spaces seen from within," in Ref. 16, pp. 79-323.

${ }^{18}$ R. Montgomery, A Tour of Sub-Riemannian Geometry, Mathematical Surveys and Monographs (American Mathematical Society, Providence, 2002).

${ }^{19}$ A. Agrachev and J. P. Gauthier, Acta Applicandae Mathematicae 57, 287 (1999).

${ }^{20}$ B. W. Shore, The Theory of Coherent Atomic Excitation (Wiley, New York, 1990), Vol. 2, p. 792.

${ }^{21}$ L. S. Pontryagin, V. Boltianski, R. Gamkrelidze and E. Mitchtchenko, The Mathematical Theory of Optimal Processes (Wiley, New York, 1961).

${ }^{22}$ V. Jurdjevic, Geometric Control Theory (Cambridge University Press, Cambridge, 1997).

${ }^{23}$ A. Agrachev, J. Dyn. Control Syst. 2, 321 (1996).

${ }^{24}$ H. Chakir, J. P. Gauthier, and I. Kupka, J. Dyn. Control Syst. 2, 359 (1996).

${ }^{25}$ C. E. Carroll and F. T. Hioe, Phys. Rev. A 42, 1522 (1990).
} 